\title{
Identification of proteins involved in response to cold stress and genome-wide identification and analysis of the APX gene family in winter rapeseed (Brassica rapa L.)
}

\section{Li Ma}

Gansu Agricultural University

Jing Bai

Zhangye Academy of Agricultural Sciences

Jia Xu

Gansu Agricultural University

Weiliang Qi

Gansu Agricultural University

Haiyun Li

Lanzhou University

\section{Yan Fang}

Gansu Agricultural University

\section{Yaozhao Xu}

Hexi University

\section{Xiucun Zeng}

Hexi University

Yuanyuan Pu

Gansu Agricultural University

\section{Wangtian Wang}

Gansu Agricultural University

\section{Lijun Liu}

Gansu Agricultural University

\section{Xuecai Li}

Gansu Agricultural University

Wancang Sun ( $\sim 18293121851 @ 163 . c o m$ )

Gansu Agricultural University

Junyan Wu ( $\nabla$ wujuny@gsau.edu.cn )

Gansu Agricultural University 


\section{Research Article}

Keywords: winter Brassica rapa, cold stress, APX gene family

Posted Date: June 7th, 2021

DOl: https://doi.org/10.21203/rs.3.rs-538668/v1

License: (c) (i) This work is licensed under a Creative Commons Attribution 4.0 International License. Read Full License 


\section{Abstract}

Winter Brassica rapa is an important oilseed crop in northern China, but the mechanism of its cold resistance remains unclear. APX plays important roles in response of this plant to abiotic stress and in scavenging free radicals. In this study, 59 DEPs were isolated and identified from winter $B$. rapa and $B$. napus using bidirectional electrophoresis, and APX was found to be differentially expressed in these two species. Therefore, the roles of APX proteins in the cold response and superoxide metabolism pathways in both rapeseed species were further investigated. And comprehensive analysis of phylogeny, chromosome distribution, motif identification, sequence structure, gene duplication, and RNA-seq expression profile in $A P X$ gene family. Most of the BrAPX genes were specifically expressed under low temperature stress and behaved significantly differently in cold-tolerant and cold-sensitive varieties. qPCR was also used to verify the differences in expression between these two varieties under cold, freezing, drought and heat stress, and these candidate genes and proteins may play important roles in the response of $B$. rapa to low temperature stress and provide new information for the elucidation of the cold resistance mechanism in $B$. rapa.

\section{Main Text}

Chilling injury is an important abiotic factor affecting crop yield, quality and growing area, regulating crop gene expression, protein levels and metabolites at the molecular level ${ }^{1}$. Proteins react directly in response to plant stress, and changes can be observed during a plant's adaptation to stress ${ }^{2}$. Mass spectrometry (MS)-based proteomics has become an important tool for unraveling the relationship between protein abundance and plant response to stress ${ }^{3,4}$. Plant antifreeze protein (AFP) is a type of active antifreeze substance that inhibits intracellular ice crystal formation and exerts an inhibitory effect on the size of ice crystals that have already formed ${ }^{5,6}$. In 1992, Griffith obtained and partially purified AFPs for the first time from atheroblasts of winter rye subjected to low temperature, pioneering the study of $\mathrm{AFPs}^{7}$. Analysis of the apoplast proteins of $B$. rapa under low-temperature stress revealed $\beta-1,3-g l u c a n a s e$, and the expression of this gene was significantly elevated under low-temperature stress ${ }^{8,9}$. This enzyme was also found to be present in AFPs of winter rye and caused ice crystals to form ${ }^{10}$. Two-dimensional electrophoresis (2-DE) and MS were used to identify a number of proteins expressed in response to $\mathrm{NaCl}$ stress in Halogeton glomeratus that are involved in pathways such as photosynthesis, carbohydrate and energy metabolism, and defense and response to adversity ${ }^{11}$. Comparative proteomic analysis of 9 developmental stages of cassava roots showed that three 14-3-3 isoforms were induced to phosphorylate during storage root expansion, and transgenic verification showed that 14-3-3 proteins and their binding enzymes play an important role in carbohydrate metabolism and starch accumulation during cassava root tuber formation ${ }^{12}$.

Cold stress leads plants to produce reactive oxygen species (ROS), including superoxide anions $\left(\mathrm{O}^{2-}\right)$, hydrogen peroxide $\left(\mathrm{H}_{2} \mathrm{O}_{2}\right)$ and hydroxyl radicals $\left(\mathrm{OH}^{-}\right)$, which destroy DNA and cellular components through lipid peroxidation and protein oxidation. Most cold-tolerant plants have evolved antioxidant 
defense mechanisms to defend against excessive accumulation of these ROS substances, including protective substances such as ascorbic acid, superoxide dismutase (SOD), catalase (CAT) and ascorbate peroxidase $(\mathrm{APX})^{13,14}$. APX belongs to the peroxidase superfamily in plants and plays an important role in ROS scavenging pathways ${ }^{15,16}$. APX is a key enzyme in the ascorbate-glutathione cycle that has evolved in plants to scavenge $\mathrm{H}_{2} \mathrm{O}_{2}$ from plant chloroplasts and the cytoplasm. It uses ascorbic acid as an electron donor to scavenge $\mathrm{H}_{2} \mathrm{O}_{2}$ produced in plants and thereby improves resistance to oxidative stress and enhances stress resistance ${ }^{17-19}$. The important role of the APX gene family in antioxidant stress has now been demonstrated in a variety of plants. Nine APX and seven GPX members have been identified in sorghum, and RNA-seq and QPCR analysis showed that APX/GPX genes were significantly regulated under drought stress ${ }^{20}$. Antioxidant and cold resistance studies on transgenic cassava coexpressing cytoplasmic MeCu/ZnSOD and MeAPX2 showed that SOD and APX expressed at high levels in transgenic plants scavenged ROS and activated antioxidant defense mechanisms, thus improving tolerance to cold stress ${ }^{21}$. A study of Arabidopsis revealed that the regulation of plastid APX could transmit information on previous cold stress over time without the establishment of cold adaptation ${ }^{22}$.Sato et al. cloned the promoter of the APXa gene from rice, found that it featured a minimum heat shock factor binding motif 5'-nGAAnnTTCn-3', located 81 bp upstream of the TATA box, and confirmed heat shock-mediated $A P X$ gene expression and protection against chilling in rice seedlings ${ }^{23}$. However, there are few studies on APX regulation of cold acclimation in winter rapeseed.

Rapeseed is one of the major oil crops in China, and the yield and oil content of winter rapeseed from northern China are higher than those of spring rapeseed and other oil crops ${ }^{24}$. Overwintering has been the main factor limiting the development of winter rapeseed. In most regions in China north of 35 degrees north latitude, extreme winter temperatures are below $-20^{\circ} \mathrm{C}$, cumulative negative air temperature is less than $-500^{\circ} \mathrm{C}$, and winter rainfall is below $30 \mathrm{~mm}^{25,26}$. These severe cold and dry conditions, combined with high winds and evaporation in the winter, cause most crops, except for winter wheat, to have difficulty overwintering ${ }^{27}$. Breeding and production of winter $B$. rapa varieties solved the problem of overwintering for winter oilseed crops and made northern cold and dry regions an important winter rapeseed production region in China, thereby increasing cropping intensity and economic return. There are significant differences in cold tolerance and field performance between winter $B$. rapa and $B$. napus. During the seedling stage, the belowground growth of $B$. rapa is vigorous, and the growth cone is buried below the soil surface. $B$. napus also exhibits vigorous aboveground growth in the seedling stage, but the growth cone is raised above the soil surface at this time, and belowground growth is less ${ }^{28,29}$. These differences in seedling growth are likely related to differences in cold tolerance ${ }^{9}$.

In this study, 59 differentially expressed proteins (DEPs) were isolated and identified in winter B. rapa and $B$. napus using the 2-DE technique, and we found that APX was differentially expressed; therefore, we performed further studies on APX proteins involved in the cold response and superoxide metabolism pathways in these two winter oilseed species. We conducted a genome-wide identification of 118 family members of APX genes in B. rapa and performed a comprehensive analysis of their phylogeny, 
chromosome distribution, motif identification, sequence structure and gene duplication. We also used RNA-seq data to identify APX genes in B. rapa and B. napus expressed in response to cold stress and qPCR data to validate the specific expression of these genes under cold stress and measured physiological indicators to further corroborate these results. This study provides information on the involvement of APX genes and their expressed proteins in the cold stress response in $B$. rapa, which will help to further elucidate the mechanism underlying strong cold resistance in $B$. rapa.

\section{Materials And Methods}

\section{Plant growth and cold treatments.}

Ultra-cold-resistant Longyou-7 (B. rapa L.) and weakly cold-resistant NTS-309 (B. napus L.) were grown in a greenhouse at Gansu Agricultural University in Gansu Province, China. A total of 120 seeds were surface-sterilized in $10 \% \mathrm{H}_{2} \mathrm{O}_{2}$ (hydrogen peroxide) for $30 \mathrm{~min}$, soaked in distilled water for $10 \mathrm{~min}$, and washed 3 times to remove $\mathrm{H}_{2} \mathrm{O}_{2}$. Seeds were germinated on two layers of wet filter paper in a glass petri dish and placed in a plant incubator $\left(22 / 18^{\circ} \mathrm{C}\right.$, day/night) for 2 days. Three uniform seedlings of each cultivar were selected, with good growth condition and consistent growth stages, and transplanted to 15$\mathrm{cm}$ diameter seedling pots filled with matrix and vermiculite (volume: volume, 3:1). At the six-leaf stage, seedlings were moved into an artificial climate chamber for low-temperature treatments at $4{ }^{\circ} \mathrm{C}$ for $24 \mathrm{~h}$. A control treatment was prepared at $22{ }^{\circ} \mathrm{C}$. The growth cone of each plant was collected after the treatment was completed, rinsed with distilled water, blotted dry on filter paper, dissected into approximately 5-mm thick slices, frozen immediately in liquid nitrogen, and subsequently stored at $-80{ }^{\circ} \mathrm{C}$ for further analysis. Three independent experiments were performed as biological replicates. Two winter $B$. rapa varieties (Longyou-7 and Lenox) were tested with qPCR while under abiotic stress. Cold, freezing and heat stress treatments were carried out in incubators at $4{ }^{\circ} \mathrm{C},-4^{\circ} \mathrm{C}$ and $40^{\circ} \mathrm{C}$, respectively. Drought stress treatment was applied by adding $200 \mathrm{ml}$ of a nutrient solution containing 18\% PEG6000 to the medium of each pot. After treatment for $0 \mathrm{~h}(\mathrm{CK}), 3 \mathrm{~h}$ and $24 \mathrm{~h}$, the growth cones and leaves were collected according to the method described above ${ }^{30}$.

\section{Measurement of physiological indicators}

Malondialdehyde (MDA) content was determined by the method of Hodges ${ }^{16}$, with some modifications; $10 \%$ trichloroacetic acid (TCA) and $0.6 \%$ thiobarbituric acid (TBA) were used to detect MDA. Plant tissue enzyme extraction was performed using precooled $0.1 \mathrm{M}$ sodium phosphate buffer $(\mathrm{pH} 7.0)$ and mortar and pestle. CAT activity was calculated by measuring the decrease in absorbance at $240 \mathrm{~nm}$ with $\mathrm{H}_{2} \mathrm{O}_{2}$ (hydrogen peroxide) ${ }^{31}$. Peroxidase (POD) activity was measured as described by Mahan ${ }^{31}$. Superoxide dismutase (SOD) was estimated according to the method developed by Gill ${ }^{32}$. Ascorbate peroxidase (APX) activity was measured using the method described by Nakano ${ }^{33}$. Soluble sugar (SS) was measured following Yemm ${ }^{34}$. The Bradford method, applying the principle of protein-dye binding, was used to determine the soluble protein (SP) content $^{35}$. 


\section{Protein extraction, two-dimensional gel electrophoresis (2-DE) and image analysis}

Total proteins were extracted according to the method described by Wang and $\mathrm{Li}^{11,36}$, with modifications. The protein concentration for each temperature treatment was determined according to the method described by Peterson, and bovine serum albumin (BSA) was used to map the protein standard curve $\mathrm{e}^{37}$.

For isoelectric focusing (IEF) in the first dimension, protein samples were dissolved in hydrated buffer with $7 \mathrm{M}$ urea, $2 \mathrm{M}$ thiourea, 4\% (w/v) CHAPS, $65 \mathrm{mM}$ DTT, 0.001\% (w/v) bromophenol blue and $0.2 \%$ (w/v) ampholytes ( $\mathrm{pH} 4-7)$ (Bio-Lyte; Bio-Rad, Hercules, CA, USA). One thousand micrograms of each protein sample was loaded into an IPG strip holder. Immobilized linear gradient strips (pH 4-7, 17 cm; BioRad) were rehydrated at $50 \mathrm{~V}$ for $14 \mathrm{~h}$ at $20^{\circ} \mathrm{C}$. Isoelectric focusing was performed using EttanTMIPGphor3 (GE Healthcare) at $20^{\circ} \mathrm{C}$ using a six-step process: $250 \mathrm{~V}$ for $1.5 \mathrm{~h}$ with a linear ramp, $500 \mathrm{~V}$ for $1.5 \mathrm{~h}$ with a linear ramp, $1000 \mathrm{~V}$ for $2.5 \mathrm{~h}$ with a celerity ramp, $10,000 \mathrm{~V}$ for $5 \mathrm{~h}$ with a linear ramp, $10,000 \mathrm{~V}$ for $92,000 \mathrm{~V}$-h with a celerity ramp, and $500 \mathrm{~V}$ for unspecified time with a celerity ramp. After IEF, the strips were incubated for $15 \mathrm{~min}$ in equilibration buffer I with $6 \mathrm{M}$ urea, 2\% sodium dodecyl sulfate (SDS), $0.375 \mathrm{M}$ Tris- $\mathrm{HCl}$ (pH 8.8), 20\% glycerol and $130 \mathrm{mM} \mathrm{DTT}$. The strips were then incubated in equilibration buffer II with $6 \mathrm{M}$ urea, $2 \%$ SDS, $0.375 \mathrm{M}$ Tris- $\mathrm{HCl}(\mathrm{pH} 8.8), 20 \%$ glycerol and $135 \mathrm{mM}$ iodoacetamide for $15 \mathrm{~min}$.

Second-dimensional separation of proteins was performed on $12.5 \%$ polyacrylamide SDS gels using GE Ettan DALTsix (GE Healthcare). Gels were stained with CBB G-250. The 2-DE gels were scanned using a UMAX PowerLook 2100XL scanner (Power Company, Chinese Taipei). A total of 12 2-DE gels were analyzed using PDQuest software (version 8.0.1, Bio-Rad). Changes greater than 2-fold in spots between control and treatment samples were analyzed. Protein spots were selected for analysis only if the protein spots were confirmed to show consistent abundance levels in three independent sample sets ${ }^{38}$.

\section{Protein identification and functional analysis}

Selectable repeatable protein spots were trypsinized for subsequent experiments ${ }^{39}$. MS and MS/MS data for protein identification were obtained by using a MALDI-TOF-TOF instrument (5800 proteomics analyzer, Applied Biosystems). Instrument parameters were set using 4000 Series Explorer software (Applied Biosystems). The MS spectra were recorded in reflector mode in a mass range from 800 to 4000 with a focus mass of 2000. TOF/TOF calibration mixtures (AB SCIEX) were used to calibrate spectra to a mass tolerance within $10 \mathrm{ppm}$. The MS spectra were processed using TOF-TOF Series Explorer software (V4.0, AB SCIEX). At least 1,000 laser shots were typically accumulated with a laser pulse rate of $400 \mathrm{~Hz}$ in MS mode, whereas in MS/MS mode, up to 2,000 laser shots were acquired with an average pulse rate of $1,000 \mathrm{~Hz}$. For MS calibration, autolysis peaks of trypsin were used as internal calibrates, and the most intense ion signals (up to 10) were selected as precursors for MS/MS acquisition, excluding the trypsin autolysis peaks and the matrix ion signals ${ }^{38,40}$. 
Peptide mass fingerprinting (PMF) and MS/MS queries were performed using the MASCOT search engine 2.2 (Matrix Science, Ltd.) embedded into GPS-Explorer Software 3.6 (Applied Biosystems) on the $B$. rapa (downloaded in June 2017, 42868 sequences) and B. napus (downloaded in June 2017, 63407 sequences) UniProt databases with the following parameter settings: 100 ppm mass accuracy, one missed trypsin cleavage allowed, carbamidomethylation set as fixed modification, oxidation of methionine allowed as variable modification, MS/MS fragment tolerance set to 0.4 Da. A GPS Explorer protein confidence index $\geq 95 \%$ was used for further manual validation 41,42 .

Functional information for the identification of proteins was obtained using the NCBI (http://www.ncbi.nlm.nih.gov/protein) and UniProt (http://www.uniprot.org) databases. Hierarchical clustering of expression profiles was performed with clustering software (version 3.0) using the selforganizing tree algorithm (SOTA). Gene ontology (GO) analysis was performed by Blast2GO (http://www.blast2go.com) using the GO annotation search tool and data from $\mathrm{NCBI}$ (http://www.ncbi.nlm.nih.gov). The cellular locations of identified proteins were predicted by PSORT (http://psort.hgc.jp/). Protein pathway analysis was performed using KEGG (http://www.genome.jp/kegg/pathway) ${ }^{43}$.

\section{Identification and analysis of peroxidase genes in B. rapa and B. napus}

The HMM (hidden Markov model) file of the peroxidase domain PF00141 was downloaded from the Pfam database (http://pfam.xfam.org/) ${ }^{44}$, and the genome and protein sequences of $B$. rapa and $B$. napus were downloaded from the Brassica database (BRAD) (http://brassicadb.org/brad/index.php) ${ }^{45}$. First, the HMM profiles corresponding to peroxidase domain sequences of $B$. rapa and $B$. napus were constructed by HMMER 3.1 software (http://hmmer.org/download.html) with an E-value $\leq 1^{\mathrm{e}-10} 46$, and then SMART software (http://smart.embl-heidelberg.de) ${ }^{47}$, NCBI-CDD (http://www.ncbi.nlm.nih.gov/Structure/cdd/wrpsb.cgi) and the Pfam database were used to remove redundant sequences and confirm the obtained peroxidase proteins ${ }^{20,48-50}$. Finally, the final sequence file was manually selected and confirmed for follow-up experiments. The basic physicochemical properties of protein sequences were analyzed using the ExPasy site (http://web.expasy.org/protparam/) ${ }^{51}$.

\section{Sequence analysis, structural identification and phylogenetic classification of BrAPX genes}

The conserved motifs in the BrAPX sequence were identified using the MEME tool (version 5.0.4, http://alternate.meme-suite.org/tools/meme) with the following parameters: the maximum number of motifs was set to 10, and the optimal motif width was 6 to 50 amino acid residues ${ }^{52}$. Exon-intron structural information for the BrAPX genes was mapped using the Gene Structure Display Server (GSDS2.0, http://gsds.cbi.pku.edu.cn/) ${ }^{53}$. Homologous sequence alignment of APX amino acid sequences identified in $B$. rapa and $B$. napus was performed using the ClustalW program ${ }^{54}$, and an unrooted phylogenetic tree was generated using MEGA (version 7.0) by the neighbor joining (NJ) method with 1000 bootstrap samples ${ }^{55,56}$. 
Analysis of chromosomal distribution and gene duplication in $B$. rapa

Chromosomal position mapping of BrAPX genes was performed using MapChart software ${ }^{57}$. Analysis and visualization of tandem duplication and segmental duplication between $B$. rapa and itself and $B$. rapa and B. napus was accomplished using the Multiple Collinearity Scan toolkit (MCscanX) (http://chibba.pgml.uga.edu/mcscan2/) and Circos (version 0.69) ${ }^{58,59}$. KaKs Calculator (version 2.0) (https://sourceforge.net/projects/kakscalculator2/) was used to calculate synonymous (Ks) and nonsynonymous (Ka) substitutions to further characterize the variation in the BrAPX genes ${ }^{60}$.

\section{RNA isolation, quantitative real-time PCR and RNA-seq analysis}

Total RNA was isolated using a SteadyPure Plant RNA Extraction Kit (Accurate Biotechnology, AG21019, Hunan, China) following the manufacturer's instructions and removing genomic DNA contamination. A spectrophotometer (NanoVueTM Plus, Wilmington, DE, USA) was used to evaluate RNA concentration and mass by determining the A260/A280 and A260/A230 ratios, respectively. First-strand cDNA was synthesized with Evo M-MLVRT Premix (Accurate Biotechnology, AG11706, Hunan, China) according to the instructions. qPCR was performed on an ABI QuantStudio 5 (Thermo, American) using the SYBR® Green Premix Pro Taq HS qPCR Kit (Accurate Biotechnology, AG11718, Hunan, China) with primers provided in Table S1. The qPCR reaction conditions were as follows: $30 \mathrm{~s}$ at $95^{\circ} \mathrm{C}$, followed by 40 cycles of $5 \mathrm{~s}$ at $95^{\circ} \mathrm{C}$ and $30 \mathrm{~s}$ at $60^{\circ} \mathrm{C}$, followed by $65-95^{\circ} \mathrm{C}$ melting curve detection. The qPCR efficiency of the genes was obtained by analyzing the standard curve of $C D N A$ gradient dilution, and the gene fragment encoding $B$. rapa $\beta$-actin RNA was used as the internal control to normalize the amount of template CDNA. Relative expression values for each gene were computed using the comparative $2^{-\triangle \Delta C T}$ method with normalization to the internal control gene ${ }^{61,62}$.

An RNA-seq library (SRP179662) was derived from growth cones of $B$. rapa cultivars Longyou-7 (coldtolerant) and Lenox (cold-sensitive) under cold stress $\left(22^{\circ} \mathrm{C} \text { as a control, and } 4{ }^{\circ} \mathrm{C} \text { for } 3 \mathrm{~h} \text { and } 24 \mathrm{~h}\right)^{63}$. Another RNA-seq library was derived from leaves of $B$. napus cultivars NTS-309 (cold-tolerant) and Tianyou-2238 (cold-sensitive) under cold stress $\left(25^{\circ} \mathrm{C}\right.$ as a control and $4{ }^{\circ} \mathrm{C}$ for $48 \mathrm{~h}$ ) ${ }^{64,65}$. RNA-seq means from the same gene in two samples were considered statistically significant and a heat map was drawn when there was a fold change greater than 2 and when the adjusted p-value was less than $0.05^{66}$.

\section{Statistical analysis}

One-way analysis of variance and Duncan's multiple range test were used to detect significant differences among the means of the plant treatment groups using SPSS 19.0 statistical software (SPSS Inc., Chicago, IL, USA). A $p$-value $\leq 0.05$ was considered statistically significant. All results are represented as the mean \pm standard error of the mean of at least three replications.

\section{Results}


Plant height, aboveground fresh weight and aboveground dry weight of Longyou-7 were lower than the values for NTS-309, but its root length, root diameter, belowground fresh weight, belowground dry weight, and root-shoot ratio were significantly greater than those of NTS-309 (Figure 1). The fresh weight and dry weight of the underground part of Longyou-7 were 1.78- and 1.94-fold greater than those of TS-309, respectively. The fresh weight and dry weight of TS-309 were 1.52- and 1.53-fold greater than those of Longyou-7, respectively. The two types of winter rapeseed differed in dry matter during the seedling stage. Nutrient accumulation of Longyou-7 seedlings was mainly in belowground tissue, and this could provide nutrition needed for overwintering. Shoots of NTS-309 seedlings grew vigorously, but belowground growth was weaker. In winter, the aboveground parts freeze, and the belowground parts store fewer nutrients, which leads to a lower overwintering ability.

\section{Analysis and comparison of the dynamic changes in physiological parameters}

Peroxidase (POD), superoxide dismutase (SOD), soluble protein (SP), ascorbate peroxidase (APX) and soluble sugar (SS) increased significantly after application of cold stress (Figure 2). Under cold stress, Longyou-7 exhibited higher POD, SOD, SP, APX and SS than did TS-309. After being subjected to cold stress, levels of catalase (CAT) and proline (Pro) increased, and these quantities were higher for Longyou7 than for TS-309. These results indicate that Longyou-7 features strong cold resistance and high ability to scavenge ROS. The content of malondialdehyde (MDA) is an important index that directly reflects damage to plant cell membranes ${ }^{67}$. During cold stress, MDA was lower for Longyou-7 than that of TS309 , indicating that the enzyme system of Longyou-7 is better able to resist cold stress and reduce damage.

\section{Clustering and comparison of changes in protein expression patterns by 2-DE in winter rapeseed under cold stress}

After sequencing mass spectrometry, 29 different protein spots were successfully matched for Longyou-7 (Figure 3, Figure S1, and Table S2-S3), with 24 samples having protein scores with confidence intervals greater than $95 \%$. The first category for Longyou-7 contained 11 high-abundance proteins expressed under cold treatment, including pathogenesis-related protein 5-like (spot 2), Kunitz-type serine protease inhibitor (spots 5, 6 and 11), L-ascorbate peroxidase 1 (spot 14), and superoxide dismutase [Cu-Zn] (spot 20). The second category contained 13 low-abundance proteins expressed under cold treatment, including probable fructokinase-1 (spot 16) and S-adenosylmethionine synthase (spot 25) (Figure 4-A). Thirty different protein spots were screened from TS-309 after sequencing mass spectrometry, and the protein score confidence intervals exceeded 95\% in 25 samples (Figure 3, Figure S1, and Table S4-S5). The first class of NTS-309 consisted of 4 proteins expressed in low abundance under cold treatment and not expressed under normal temperature treatment Kunitz trypsin inhibitor 1-like (spots 24, 30), and RNAbinding protein CP29B (spots 2,9) not expressed under cold treatment. The second category contained 17 high-abundance proteins expressed under cold treatment, including bifunctional enolase 2/transcriptional activator (spot 6), L-ascorbate peroxidase 1 (spot 15, 25), superoxide dismutase [Cu-Zn] (spot 18), glutathione S-transferase DHAR1 (spot 27), and Kunitz-type serine protease inhibitor (spots 11, 
13, and 19) (Figure 4-C). The expression levels of the proteins detected in the low temperature treatment were compared, indicating that APX, PR, KTSPI and SOD appeared in higher abundance as protein spots after cold stress in Longyou-7.

\section{Identification and functional pathway analysis in winter rapeseed under cold stress}

Proteins detected in Longyou-7 were classified into three categories: biological processes, molecular function, and cellular components, which accounted for $33.64,33.64$, and $32.72 \%$ of the total, respectively (Figure $4 \mathrm{~A}-\mathrm{C}$ ). Results of mass spectrometric protein analysis showed that soluble inorganic pyrophosphatase 6 (spot 7), L-ascorbate peroxidase 1 (spot 14), and S-adenosylmethionine synthase (spot 25) participate in the response to temperature stimulus pathway, and the Kunitz-type serine protease inhibitor DrTI-like (spot 5) is involved in the response to hydrogen peroxide pathway. Pathogenesis-related protein 5-like (spot 2) participates in the defense response, superoxide dismutase [Cu-Zn] (spot 20) is related to the removal of superoxide radical pathway, and fructose kinase-1 (spot 16) is involved in the glucan metabolic process.

Proteins detected in NTS-309 were classified into three categories: biological processes accounted for $33.64 \%$, molecular function accounted for $36.36 \%$ and cellular components accounted for $30 \%$ (Figure 4 D-F). Results of mass spectrometric protein analysis showed that RNA-binding protein CP29B (spot 2), bifunctional enolase 2/transcriptional activator (spot 6), Kunitz trypsin inhibitor 1-like (spot 24), and Lascorbate peroxidase 1 (spot 15) are involved in the response to cold pathway. Superoxide dismutase [Cu-Zn] (spot 18) was found to be involved in the superoxide metabolic process, and glutathione Stransferase (spot 27) was found to participate in the L-ascorbic acid metabolic process.

Using KEGG pathway identification of proteins retrieved from the database, the 19 proteins from Longyou-7 were annotated and were found to be involved in 19 metabolic pathways. The 29 proteins identified in NTS-309 were annotated and were involved in 18 metabolic pathways (Figure 5 A-B). Venn plot analysis illustrates the number of differentially expressed proteins and the overlap of these proteins between two cultivars of winter oilseed. Six common proteins were detected under cold stress in two types of winter oilseed: cysteine protease inhibitor WSCP, nucleoside diphosphate kinase 1, L-ascorbate peroxidase, superoxide dismutase [Cu-Zn], chloroplast ribulose 1,5-bisphosphate carboxylase/oxygenase small subunit and CBS domain-containing protein CBSX3 (Figure $5 \mathrm{C}$ ).

\section{Identification, chromosomal distribution, and classification of BrAPX genes}

The identification of $A P X$ genes in $B$. rapa and $B$. napus was completed using HMM searches with the aid of the BRAD genome database. These proteins were identified as having the reported peroxidase domains after sequence analysis using the SMART, CDD, Pfam and InterProScan tools. Finally, 118 and 221 candidate $A P X$ genes were obtained for $B$. rapa and $B$. napus, respectively. BrAPX amino acid residue lengths ranged from 250 aa (Bra030706) to 723 aa (Bra011683), isoelectric point (pl) values ranged from 4.40 (Bra036445) to 10.78 (Bra019132), and molecular weights ranged from $27.39 \mathrm{kDa}$ (Bra017830) to $80.97 \mathrm{kDa}$ (Bra011683) (Table S6). We mapped the chromosomal locations of the APX genes in B. rapa 
(Figure 6), with most genes located on chromosomes A1 (16 genes), A2 (18 genes), A3 (18 genes) and A9 (15 genes), followed by chromosome A10 (12 genes) and chromosomes A4, A5, A6, A7 and A8 with 7, 6, 9, 10 and 7 genes, respectively, indicating that $A P X$ genes are more widely distributed in the $B$. rapa genome.

To further investigate the evolutionary relationship of APX proteins in $B$. napus and $B$. rapa, a rootless neighbor-joining (NJ) phylogenetic tree was constructed with APX protein sequences of $B$. napus and $B$. rapa to explore the reasons for their cold resistance (Figure 7). APX was clustered into 13 subfamilies (IXIII) based on sequence similarity and topological structure. Subfamilies IV and VIII contain only 8 and 6 family members; in particular, two proteins identified by our mass spectrometry (Bra018677 and BnaC05g05550D) were clustered in the same subfamily. In addition, we subjected the 118 BrAPX members to unrooted developmental tree construction, dividing these members into 14 subclasses, with cluster $\mathrm{X}$ and cluster XIII each containing one member (Figure 8-A).

\section{Conserved motifs and structure of BrAPX genes}

Intron-exon structural models of the $A P X$ genes were generated through the GSDS server and revealed that the numbers of introns and exons in the $A P X$ gene were 10-1 and 9-0, respectively (Figure 8-B and Table S6). Subfamily XIV had the highest number of introns (Bra015668, 9; Bra013053, 8; Bra017686, 8; Bra030706, 7; Bra018677 8; Bra031598, 5), subfamily XIl had the lowest number of introns (Bra039059, 0; Bra033040, 0; Bra025604, 0; Bra024635, 0; Bra040175, 0; Bra022090, 0; Bra024972, 1), and subfamily III contained nine introns in the gene (Bra011683). The exon-intron structures of these genes distributed in the same cluster are highly conserved.

The MEME server was used to predict the motifs of BrAPX proteins, and a total of 10 widely-distributed conserved motifs were identified (Figure 8-C). Motif 2, motif 4, and motif 6 were widely present in 118 APX proteins, with motif 2 containing 50 amino acids, motif 4 containing 48 amino acids, and motif 6 containing 41 amino acids, indicating that these motifs have been functionally conserved during evolution (Table S7). Also, motif 1 and motif 7 were also present in half of the APX proteins, containing 42 and 29 amino acids, respectively. This suggests that these motifs represent conserved motifs and functional domains of the APX protein family in B. rapa.

Conserved motif distribution of BrAPX proteins; differently colored boxes represent the 10 conserved domains identified.

\section{Gene duplication and genomic collinearity in B. rapa}

Tandem duplication and segmental duplication events in the B. rapa and B. napus genomes were studied using MCScanX software (Figure 9 and Table S8). No tandem duplication events were identified in the $B$. rapa and $B$. napus $A P X$ gene families; subsequently, we identified 62 segmental duplications in $B$. rapa and 193 segmental duplications in $B$. napus, and these segmental duplications were in 90 genes each in the $B$. napus $\mathrm{A}$ and $\mathrm{B}$ genomes. These results suggest that $A P X$ genes may have arisen through gene 
duplication and that segmental duplication events have played an important role in their evolution. To further explore the potential evolutionary processes of the $A P X$ gene family in Brassica, a covariance map of $B$. rapa and $B$. napus was constructed, showing that 268 APX homologous sequences are shared between $B$. rapa and $B$. napus, suggesting that there may be substantial similarities in the molecular functions of $A P X$ between the two species and that differences in resistance may be due to differences in a few genes. In addition, we calculated $\mathrm{Ka} / \mathrm{Ks}$ values for BrAPX genes, and all pairs of segmentally duplicated $B r A P X$ genes had $\mathrm{Ka} / \mathrm{Ks}$ values $<1$, indicating that most of these genes evolved under purifying selection (Figure 9-D and Table S8).

\section{Expression of APX genes in B. rapa and B. napus under cold stress}

To investigate the expression patterns of BrAPX genes under cold stress, RNA-seq expression in growth cones were analyzed for B. rapa varieties differing in cold resistance, and heatmaps were drawn based on their protein evolutionary relationships (Figure 10-A and Table S9). The results showed that the five BrAPX genes (Bra003918, Bra017120, Bra022195, Bra001084, and Bra024268) were highly expressed in cold-tolerant varieties and were more highly expressed at $24 \mathrm{~h}$ than at $3 \mathrm{~h}$ of cold stress but were all downregulated in cold-sensitive varieties. Expression levels of the four genes (Bra012061, Bra040175, Bra026470, Bra039872) were higher in the cold-sensitive variety than in the cold-tolerant variety at $24 \mathrm{~h}$ of cold stress. There were 21 and 12 genes significantly upregulated in cold-tolerant varieties at $3 \mathrm{~h}$ and $24 \mathrm{~h}$ under cold stress, respectively, and 8 and 5 genes upregulated in cold-sensitive varieties at $3 \mathrm{~h}$ and $24 \mathrm{~h}$ under cold stress, respectively. Most of the BrAPX genes were downregulated or not significantly expressed. Similarly, we selected cold-tolerant and cold-sensitive winter $B$. napus RNA-seq expression profiles under cold stress to further analyze the expression patterns of $B n A P X$ genes. We used the same method to draw the expression heatmap of $B n A P X$ for leaves under cold stress (Figure 10-B and Table S9). The results showed that the expression of 28 genes was significantly higher in cold-tolerant varieties than in cold-sensitive varieties at $24 \mathrm{~h}$ of cold stress and that some genes (BnaC03g28220D, BnaA02g02520D, and BnaC09g48860D) were significantly upregulated in cold-tolerant varieties and significantly downregulated in cold-sensitive varieties. The expression of 48 genes was significantly higher in cold-sensitive varieties than in cold-tolerant varieties at $24 \mathrm{~h}$ of cold stress, and 38 of these genes were significantly upregulated in cold-sensitive varieties and significantly downregulated in coldtolerant varieties. In addition, we found that most $A P X$ genes were downregulated in winter $B$. napus.

We selected 10 differentially expressed BrAPX genes from the RNA-seq data for qPCR analysis and showed that the GPCR expression pattern of BrAPX genes in winter $B$. rapa growth cones under cold stress was consistent with the RNA-Seq dataset with high linearity $\left(y=1.029 x+0.241, R^{2}=0.876\right)$. In addition, the expression patterns of the 10 BrAPX genes in winter $B$. rapa leaves under cold stress tended to be consistent with those in growth cones (Figure 11).

\section{Expression patterns of APX genes in B. rapa under abiotic stress}


To further investigate the expression pattern of these $10 A P X$ genes differentially expressed under cold stress under other abiotic stresses, we performed qPCR analysis on these two varieties under heat $\left(40{ }^{\circ} \mathrm{C}\right)$, PEG (PEG6000, 18\%), and freezing $\left(-4^{\circ} \mathrm{C}\right)$ stress conditions for $3 \mathrm{~h}$ and $24 \mathrm{~h}$ (Figure 12). Five APX genes (Bra035235, Bra003918, Bra033040, Bra017120, and Bra031934) were significantly expressed in cold-tolerant varieties under freezing stress and were more highly expressed than in cold-sensitive varieties. The expression of these genes was higher in growth cones than in leaves, suggesting that coldtolerant varieties of growth cones have an important role in cold resistance. When leaves wilted after the cold overwintering period in northern China, these results were also shown during cold stress. After $24 \mathrm{~h}$ of simulated drought stress, Bra035235 and Bra017120 were significantly upregulated in Longyou-7 and Lenox growth cones, and levels were higher in Longyou-7 than in Lenox, while Bra031934 was significantly upregulated in Longyou-7 growth cones and not significantly expressed in Lenox. Under heat stress, Bra035235, Bra003918, Bra015403, Bra033040 and Bra031934 were significantly upregulated in growth cones and leaves of both species, with higher expression in Longyou-7 than in Lenox. The expression of the Bra017120 gene in Longyou-7 growth cones was higher than that in Lenox growth cones. The results showed that winter $B$. rapa leaves were more susceptible to heat stress, and coldtolerant varieties may additionally have stronger heat tolerance. In summary, the Bra035235, Bra003918, Bra033040, Bra017120 and Bra031934 genes are closely related to the abiotic stress response in winter B. rapa and deserve further study.

\section{Discussion}

Overwintering determines the cold resistance of winter rapeseed, and the high wintering capability of $B$. rapa is reflected in growth cone depression during seedling growth. Compared with $B$. napus, $B$. rapa is better able to resist the damage and death caused by extremely low temperature weather ${ }^{64}$. Due to the damage and death caused by the weather, the growth cone of $B$. napus is prominent, and the seedlings stand upright. In winter, in windy weather, stems can easily be killed by frostbite, resulting in reduced yields. During vegetative growth stages, dry matter accumulation of $B$. rapa was mainly concentrated belowground, photosynthetic organic products were preferentially distributed and transported belowground, and the aboveground portion of winter rapeseed died during the overwintering period. The metabolic energy needed for overwintering is supplied by roots, and sufficient dry matter accumulation by the strong cold-resistant varieties guarantees availability of energy for winter ${ }^{65}$. These cold conditions produce ROS that are controlled and scavenged by $A P X$ family genes such as $A P X$, which form the first line of defense against ROS. APX is known to be involved in a variety of abiotic stress-related responses, but information for the complete characterization of the $A P X$ gene family in $B$. rapa has not yet been reported. The availability of a study of the whole genome sequence description of $A P X$ in $B$. rapa could help further explain the mechanism underlying its strong cold resistance and be further used to improve cold resistance and field yields in other winter crops, such as B. napus.

\section{The $A P X$ gene family contains important information on the evolution and origin of $B$. rapa}


APX (EC, 1.11.1.11) belongs to the peroxidase (PF00141) family, is considered to be a key enzyme in $\mathrm{H}_{2} \mathrm{O}_{2}$ elimination, and plays a critical role in plant growth and development and in response to adversity ${ }^{14,68}$. This class I peroxidase catalyzes the conversion of $\mathrm{H}_{2} \mathrm{O}_{2}$ to $\mathrm{H}_{2} \mathrm{O}$ and $\mathrm{O}_{2}$ using ascorbic acid as a specific electron donor ${ }^{69}$. The detoxification of $\mathrm{H}_{2} \mathrm{O}_{2}$ by APX is a series of reactions catalyzed by monodehydroascorbate reductase (MDHAR), dehydroascorbate reductase (DHAR) and glutathione reductase (GR). Together, these reactions form one of the most important antioxidant systems in plants, the ascorbate-glutathione or Halliwell-ASADA cycle. In this cycle, ascorbate and glutathione are used as reducing substrates for the scavenging of $\mathrm{H}_{2} \mathrm{O}_{2}$ and are ultimately cycled at the expense of ATP and $\mathrm{NAD}(\mathrm{P}) \mathrm{H}^{70}$. According to the phylogeny of 74 reported $A P X$ members in many plants, they can be divided into four subfamilies, and the subcellular localization of these members may be different ${ }^{71}$. Genomewide identification of APX has now been carried out in crops such as sorghum and cotton and has provided information on the responses of some members to abiotic stresses ${ }^{20,68}$. In this study, 118 APX genes were identified in $B$. rapa, and comparison of the results with the B. napus APX gene family letters revealed that the $A$ genome had significantly fewer APX members than did the $A$ and $C$ genomes and that APX is closely linked evolutionarily between these two species ${ }^{72}$. These genes in $B$. rapa were divided into 14 subfamilies with highly consistent phylogenetic classification and intron-exon structural information (Figure 8), with low homology and functional domains in the different APX subfamilies, indicating that the BrAPX families are highly divergent in origin and functional evolution, similar to the findings in cotton.

Tandem duplication and segmental duplication contribute to the expansion of new gene family members and novel functions in the evolution of plant genomes ${ }^{73,74}$. The results of this study show that there have been no tandem duplication events in the $A P X$ gene family of $B$. rapa, 62 segmental duplication events have occurred, and that most of the BrAPX gene segmental duplications are clustered on chromosomes A1, A2, and A3. In B. napus, we also found greater numbers of segmental duplications on chromosomes $\mathrm{A} 1, \mathrm{~A} 2$, and $\mathrm{A} 3$. This suggests that segmental duplication events have played an important role in the evolution of the BrAPX genes ${ }^{66}$. As a result of duplication events, some members have been able to evolve to acquire new functions to enhance their resistance or have become pseudogenes and lost their original functions ${ }^{75}$. Gene duplication has built a large library of functional genes, enabling $B$. rapa to adapt to different environments.

\section{The peroxidase family of winter $B$. rapa responded to cold stress}

Our previous studies have shown that the expression of peroxisome pathway genes in different cold-resistant varieties of $B$. rapa differs under cold stress, and these differentially expressed genes are closely related to cold resistance ${ }^{63,65}$. This study further analyzed the expression of the APX gene family under cold stress. At 3 and 24 h of cold stress, Bra003918, Bra017120, Bra022195, Bra001084, and Bra024268 were significantly upregulated in the cold-tolerant variety Longyou-7, with Bra003918 3.71 and 4.32 times more highly expressed than in the weakly cold-resistant variety Lenox, respectively (log2fold), whereas it was not significantly expressed in Lenox. (Figure 10-A and Table S9). We also found that 21 of the 45 upregulated genes were significantly expressed in cold-tolerant varieties at $3 \mathrm{~h}$ of cold stress, 
and most of them were expressed more than in weakly cold-resistant varieties. Although the downregulated genes in both cases were slightly different among individuals, the number of downregulated genes was similar, and the number of differentially downregulated genes was much greater than that of upregulated genes. This suggests that $A P X$ is activated early in cold stress, allowing cold-tolerant varieties to establish defenses against $\mathrm{H}_{2} \mathrm{O}_{2}$ as early as possible ${ }^{76,77}$. Teixeira et al. revealed the functional diversity of the $A P X$ gene in rice, pointing to its complementarity and coordination of antioxidant defenses in different cellular compartments during developmental and abiotic stresses ${ }^{70}$. These upregulated genes were distributed across 10 subclusters, with subcluster VIII having a higher number of upregulated genes (Figure 8-A), which may be related to the functional diversity of the rape $A P X$ family and the ability of these plants to cope with different environmental challenges ${ }^{78}$.

Drought has been reported to induce upregulation of $A P X$ gene expression patterns in sorghum leaves and downregulation of the $A P X$ gene in roots, with most differentially expressed genes located in chloroplasts, mitochondria and peroxisomes ${ }^{20}$. The analysis showed that drought stress caused stomatal closure to reduce water loss and reduce the content of $\mathrm{CO}_{2}$ in leaves, thus promoting the oxidation of RuBP and increasing the content of $\mathrm{H}_{2} \mathrm{O}_{2}$. The increase in photorespiration associated with low carbon dioxide increases the electron pressure in mitochondria, thus promoting the production of reactive oxygen species ${ }^{79,80}$. Since reactive oxygen species such as $\mathrm{H}_{2} \mathrm{O}_{2}$ are the main targets of APX and GPX enzymes, it is reasonable to expect them to increase activity under drought stress. The main reason for the decrease in $A P X / G P X$ expression in sorghum roots may be the change in the source-sink relationship, and it has been noted that these differentially expressed genes may play an important role in alleviating drought stress in sorghum ${ }^{20}$. APX was determined in this study to be differentially upregulated, but its corresponding mRNA (Bra018677) expression was not significant. APX metabolism forms a complex network, and the concentration of a metabolite is regulated by the interaction of multiple genes. Studies have shown that cotton GhAPX genes may perform multiple functions in the regulation of cotton fiber development by $\mathrm{H}_{2} \mathrm{O}_{2}$ and phytohormones and that there is also a phenotype consisting of duplicate enantiomers displaying different response profiles, implying their functional and regulatory diversity ${ }^{68}$. Other studies have shown that $A P X$ transgenic plants of Arabidopsis, potato, sweet potato, cotton and other crops may perform a variety of functions during abiotic stress, with generally elevated APX content and enhanced ability to eliminate reactive oxygen species ${ }^{76,81-83}$. In the present study, we were surprised to find that the Bra035235, Bra017120 and Bra031934 genes were significantly upregulated under all four abiotic stress conditions, and this upregulation trend was more prominent in the cold-tolerant varieties. It is well known that winter $B$. rapa is widely grown in northern China, and the ecological conditions in this region are cruel. Winter $B$. rapa is subject to various abiotic adversities throughout the reproductive period, and varieties with strong cold tolerance tend to display other types of abiotic resistanc ${ }^{26,30}$. However, individual genes show opposite results under different abiotic stresses, implying the complexity of the response of winter B. rapa to abiotic stresses.

\section{Antioxidant proteins play an important role in the cold resistance of winter rapeseed}


After plants are subjected to cold stress, ROS accumulates and destroys the structure of cells ${ }^{15}$. In severe cases, the excessive loss of water by cells leads to the death of the leaves ${ }^{84}$. At this point, the strength of a plant's antioxidant capacity is directly reflected in its ability to return the generated ROS to normal levels ${ }^{85}$. In Longyou-7, superoxide dismutase [Cu-Zn] (spot 20) was involved in removal of superoxide radicals, Kunitz-type serine protease inhibitor DrTI-like (spots 5, 6, and 11) was related to response to hydrogen peroxide, L-ascorbate peroxidase 1 (spot 14) was involved in the L-ascorbic acid metabolic process, and protein synthesis was upregulated for all of these genes under cold stress. We also measured the activities of APX and SOD, which also increased significantly at low temperature. In TS-309, superoxide dismutase [Cu-Zn] (spot 18) and glutathione S-transferase DHAR1 (spot 27) participate in the response to superoxide, L-ascorbate peroxidase 1 (spot 15 and 25) participates in the L-ascorbic acid metabolic process, and Kunitz-type serine protease inhibitor DrTI-like (spot 11, 19, 24, and 30) is related to the response to hydrogen peroxide. These proteins are upregulated or exhibit new protein spots under cold stress. The results show that cold stress induced the expression of antioxidant enzyme genes in $B$. napus and $B$. rapa and increased antioxidant protein and enzyme activity. However, the expression and activity of antioxidant enzyme genes were higher in $B$. rapa than in $B$. napus after cold treatment, which may be one of the reasons why $B$. rapa shows better cold resistance than $B$. napus. However, antioxidant proteins of $B$. napus did not show lower expression abundance than those of $B$. rapa. Perhaps $B$. rapa is more cold-resistant to certain kinds of antifreeze proteins.

At the physiological level, winter rape uses protective enzymes and regulatory substances to improve its cold resistance. After winter rapeseed leaves were subjected to low temperature stress, oxygen free radicals in the cells increased and cell structure was destroyed, and when the cells lost too much water, the leaves died. At this time, protective enzymes are increasing, and oxygen free radicals in the cells are effectively removed over time to alleviate damage caused by low temperature ${ }^{85,86}$. Increasing the content of soluble protein reduces the damage caused by water loss from cells ${ }^{87}$. The content of soluble protein in winter wheat at low temperature was positively correlated with its cold resistance ${ }^{88}$. Similarly, Zhang reported a parallel relationship between cold resistance and the content of soluble sugar in plants ${ }^{89}$. Cold injury forces membrane lipid peroxidation; increasing MDA accelerates membrane lipid peroxidation, leading to leakage of electrolytes and reduction of cellular water potential, thereby destroying normal metabolism in vivo ${ }^{90}$. APX can react to changes by transforming $\mathrm{H}_{2} \mathrm{O}_{2}$ into water and plays an important role in removing ROS produced under environmental pressure ${ }^{91}$. The results of this study also showed that protective enzymes, soluble substances, Pro and APX in winter Brassica leaves were elevated under cold stress. The physiological condition the strongly cold-resistant Longyou-7 was better than that of the weakly cold-resistant NTS-309, and the accumulation of MDA was lower.

\section{Proteins associated with the response to cold pathway}

Proteomics has been widely used in the study of plant responses to low-temperature stress and has identified many proteins associated with cold stress in sea buckthorn, Physcomitrella patens, wheat and rice $^{92-95}$. Cold shock initially increases cold resistance in most plants at nonfreezing temperatures; that 
is, during the process of cold stress, cold resistance increases ${ }^{96}$. Some of these accumulated proteins have already been under cold stress and are resistant to cold stress. SAM regulates ethylene synthesis, changes the ethylene, polyamine and cyanide contents and affects plant stress resistance ${ }^{38}$. DNA methylation and histone methylation modification also affect a plant's ability to resist environmental stress ${ }^{97}$. Studies have shown that overexpression of the SAM gene in wild soybean increased resistance to low temperature, drought, and salt tolerance in transgenic tobacco ${ }^{98}$. Plant pathogenesis-related (PR) genes are generally induced to undergo transcriptional upregulation during pathogen infection ${ }^{99}$. At present, there is evidence that PR genes also play an important role in the plant response to abiotic stress $^{100}$. Studies have shown that RNA-binding proteins play an important role in the cold acclimation of plants. Arabidopsis GRP2-and RZ-1a-overexpressing strains have clear resistance to low-temperature ${ }^{101}$. In this study, S-adenosylmethionine synthase (spot 25) was involved in the response to stress, pathogenesis-related protein (spot 2) was involved in defense, and probable fructokinase (spot 16) was involved in cellular glucan metabolic processes in Longyou-7. NTS-309 mass spectrometry revealed that RNA-binding protein CP29B (spots 2 and 9) is involved in cold acclimation, and a bio-functional enolase $2 /$ transcriptional activator (spot 6) participates in the response to the temperature stimulus.

\section{Photosynthesis under cold stress}

Chloroplast triosephosphate isomerase (spot 15), ribulose-1,5-bisphosphate carboxylase/oxygenase small subunit (spots 22 and 23), and ribulose-1,5-bisphosphate carboxylase/oxygenase large subunit (spot 26) are involved in photosynthesis in Longyou-7. This study found that spots 15, 23, and 26 were inhibited after cold stress, indicating that the photosynthetic apparatus of Longyou-7 was damaged in a low-temperature environment and that photosynthetic proton transmission and photosynthetic phosphorylation were inhibited by low temperature. In NTS-309, oxygen-evolving enhancer protein spot 8 and chloroplast ribulose-1,5-bisphosphate carboxylase/oxygenase small subunit (spots 17, 23, and 26) were found to be involved in photosynthesis. After cold stress, Rubisco protein expression was enhanced. Rubisco controls light flux through the photosynthesis carbon reduction cycle to respond to short-term changes in the environment. B. napus can normally carry out photosynthesis under cold stress, which may be related to the strong growth of shoots during the seedling stage. When plants are subjected to low-temperature signals, they can still maintain normal photosynthesis. Thus, when the belowground portion of the plant is small at the onset of winter, leaves and roots will freeze to death in winter. B. rapa features small shoots at the seedling stage and strong belowground growth, providing stable energy for overwintering $^{29}$. B. napus and $B$. rapa are regulated by different cold resistance mechanisms ${ }^{28}$.

\section{Conclusions}

In this study, 24 and 25 DEPs were identified by bidirectional electrophoresis in B. rapa and B. napus, respectively. GO analysis showed that these proteins are involved in the low-temperature response, transcriptional regulation, ion stability, photosynthesis and ROS scavenging enzyme pathways, and APX proteins were identified by their differential expression. We then identified 118 BrAPX genes from B. rapa 
and localized them unevenly to 10 chromosomes. The BrAPX proteins were classified into 14 subclasses based on their conserved structural domains and evolutionary relationships and were closely linked evolutionarily to $B$. napus, with segmental replication events playing a major role. More than half of the BrAPX genes were specifically expressed under low-temperature stress and differed significantly among varieties in cold resistance. We investigated the expression patterns of BrAPX genes in growth cones and leaves of cold-tolerant and cold-sensitive varieties under freezing, drought and heat stress and identified five key genes that respond to abiotic stresses. In addition, we identified a number of candidate genes and candidate proteins that may play important roles in the response of $B$. rapa to low-temperature stress. This work provides new ideas for the study of the involvement of the APX family in the molecular mechanisms of cold resistance in winter B. rapa.

\section{Declarations}

We wish to thank Dr. Zhen Liu, Yuhong Zhao, Xiaolei Tao, Bailin Sun, Wanpeng Wang of Gansu Agricultural University for help with the experiments. This study was funded by Research Program Sponsored by Gansu Provincial Key Laboratory of Aridland Crop Science Gansu Agricultural University (GSCS-2020-06), the Scientific research start-up funds for openly-recuited doctors of Gansu Agricultural University (GAU-KYQD-2019-17), and the China Agriculture Research System of MOF and MARA (CARS12).

\section{Author Contributions}

L.M., W.S. and J.W. conceived and designed the study. L.M., J.B., J.X .and W.Q. conducted experiments. H.L., Y.X., X.Z. and Y.F. analyzed the data. W.W., L.L., X.L. and Y.P. contributed reagents/materials/analysis tools. L.M. wrote the paper. W.S. revised the manuscript. All authors have read and approved the final version.

\section{Competing Interests}

The authors declared no competing interests.

\section{References}

1. Li, L., Lu, X., Ma, H. \& Lyu, D. Comparative proteomic analysis reveals the roots response to low rootzone temperature in Malus baccata. J. Plant Res. 131, 865-878 (2018).

2. Xuan, J. et al. Comparative Proteomic Analysis of the Stolon Cold Stress Response between the C4 Perennial Grass Species Zoysia japonica and Zoysia metrella. PLoS One 8, e75705 (2013).

3. Giege, P. Enzymes of Glycolysis Are Functionally Associated with the Mitochondrion in Arabidopsis Cells. Plant Cell 15, 2140-2151 (2003).

4. Bae, M. S., Cho, E. J., Choi, E. Y. \& Park, O. K. Analysis of the Arabidopsis nuclear proteome and its response to cold stress. Plant J. 36, 652-663 (2003). 
5. Bredow, M., Vanderbeld, B. \& Walker, V. K. Ice-binding proteins confer freezing tolerance in transgenic Arabidopsis thaliana. Plant Biotechnol. J. 15, 68-81 (2017).

6. Ding, Y. et al. EGR 2 phosphatase regulates OST 1 kinase activity and freezing tolerance in Arabidopsis. EMBO J. 38, 1-17 (2019).

7. Griffith, M., Ala, P., Yang, D. S. C., Hon, W. \& Moffatt, B. A. Antifreeze protein produced endogenously in winter rye leaves. Plant Physiol. 100, 593-596 (1992).

8. Ma, L. et al. Expression Analysis of $\beta-1,3-G l u c a n a s e ~ G e n e$ from Winter Brassica rapa Under Low Temperature Stress. Chinese Bull. Bot. 52, 568-578 (2017).

9. Liu, Z. et al. Separation and identification of Brassica rapa BrAFP and its gene cloning and expression under freezing stress. Plant Breed. 138, 193-201 (2019).

10. Hon, W., Criffith, M., Mlynarz, A., Kwok, Y. C. \& Yang, D. S. C. Antifreeze Proteins in Winter Rye Are Similar to Pathogenesis-Related Proteins. Plant Physiol. 109, 879-889 (1995).

11. Wang, J. et al. Physiological and proteomic analyses of salt stress response in the halophyte Halogeton glomeratus. Plant, Cell Environ. 38, 655-669 (2015).

12. Wang, X. et al. Proteomics Profiling Reveals Carbohydrate Metabolic Enzymes and 14-3-3 Proteins Play Important Roles for Starch Accumulation during Cassava Root Tuberization. Sci. Rep. 6, 1-15 (2016).

13. Hernández, J. A., Ferrer, M. A., Jiménez, A., Barceló, A. R. \& Sevilla, F. Antioxidant systems and 02--/ $\mathrm{H} 2 \mathrm{O} 2$ production in the apoplast of pea leaves. Its relation with salt-induced necrotic lesions in minor veins. Plant Physiol. 127, 817-831 (2001).

14. Gilroy, S. et al. ROS, calcium, and electric signals: Key mediators of rapid systemic signaling in plants. Plant Physiol. 171, 1606-1615 (2016).

15. Apel, K. \& Hirt, H. REACTIVE OXYGEN SPECIES: Metabolism, Oxidative Stress, and Signal Transduction. Annu. Rev. Plant Biol. 55, 373-399 (2004).

16. Hodges, D. M., DeLong, J. M., Forney, C. F. \& Prange, R. K. Improving the thiobarbituric acid-reactivesubstances assay for estimating lipid peroxidation in plant tissues containing anthocyanin and other interfering compounds. Planta 207, 604-611 (1999).

17. Miura, K. \& Furumoto, T. Cold signaling and cold response in plants. Int. J. Mol. Sci. 14, 5312-5337 (2013).

18. Winfield, M. O., Lu, C., Wilson, I. D., Coghill, J. A. \& Edwards, K. J. Plant responses to cold: transcriptome analysis of wheat. Plant Biotechnology Journal 8, 749-771 (2010).

19. Ding, Y., Shi, Y. \& Yang, S. Advances and challenges in uncovering cold tolerance regulatory mechanisms in plants. New Phytol. 1, 1690-1704 (2019).

20. Akbudak, M. A., Filiz, E., Vatansever, R. \& Kontbay, K. Genome-Wide Identification and Expression Profiling of Ascorbate Peroxidase (APX) and Glutathione Peroxidase (GPX) Genes Under Drought Stress in Sorghum (Sorghum bicolor L.). J. Plant Growth Regul. 37, 925-936 (2018). 
21. Xu, J., Yang, J., Duan, X., Jiang, Y. \& Zhang, P. Increased expression of native cytosolic Cu/Zn superoxide dismutase and ascorbate peroxidase improves tolerance to oxidative and chilling stresses in cassava (Manihot esculenta Crantz). BMC Plant Biol. 14, 208 (2014).

22. van Buer, J., Cvetkovic, J. \& Baier, M. Cold regulation of plastid ascorbate peroxidases serves as a priming hub controlling ROS signaling in Arabidopsis thaliana. BMC Plant Biol. 16, 1-20 (2016).

23. Sato, Y. et al. Heat shock-mediated APX gene expression and protection against chilling injury in rice seedlings. J. Exp. Bot. 52, 145-151 (2001).

24. Sun, W. et al. Study on Adaptation and Introduction Possibility of Winter Rapeseed to Dry and Cold Areas in Northwest China. Sci. Agric. Sin. 40, 2716-2726 (2007).

25. Sun, W. et al. Critical Index Analysis of Safe Over-wintering Rate of Winter Rapeseed (Bras- sica rapa) in Cold and Arid Region in North China. Acta Agron. Sin. 42, 609-618 (2016).

26. Ma, L. et al. Ecological and economic benefits of planting winter rapeseed (Brassica rapa L.) in the wind erosion area of northern China. Sci. Rep. 9, 20272 (2019).

27. Cai, J. \& Jiang, D. The Effect of Climate Change on Winter Wheat Production in China. J. AgroEnvironment Sci. 30, 1726-1733 (2011).

28. Zeng, X. et al. Identification of cold stress responsive microRNAs in two winter turnip rape (Brassica rapa L.) by high throughput sequencing. BMC Plant Biol. 18, 1-13 (2018).

29. Liu, Z. G. et al. Effects of low nocturnal temperature on photosynthetic characteristics and chloroplast ultrastructure of winter rapeseed. Russ. J. Plant Physiol. 63, 451-460 (2016).

30. Ma, L. et al. Screening and verification of reference genes for analysis of gene expression in winter rapeseed (Brassica rapa L.) under abiotic stress. PLoS One 15, e0236577 (2020).

31. Jr, B. R. \& Sizer, I. W. A spectrophotometric method for measuring the breakdown of hydrogen peroxide by catalase. J. Biol. Chem. 195, 133-140 (1952).

32. Gill, T., Sreenivasulu, Y., Kumar, S. \& Singh Ahuja, P. Over-expression of superoxide dismutase exhibits lignification of vascular structures in Arabidopsis thaliana. J. Plant Physiol. 167, 757-760 (2010).

33. Nakano, Y. \& Asada, K. Hydrogen peroxide is scavenged by ascorbate-specific peroxidase in spinach chloroplasts. Plant cell Physiol. 22, 867-880 (1981).

34. Yemm, E. W. \& Willis, A. J. The estimation of carbohydrates in plant extracts by anthrone. Biochem. J. 57, 508 (1954).

35. Bradford, M. M. A rapid and sensitive method for the quantitation of microgram quantities of protein utilizing the principle of protein-dye binding. Anal. Biochem. 72, 248-254 (1976).

36. Li, W., Zhang, C., Lu, Q., Wen, X. \& Lu, C. The combined effect of salt stress and heat shock on proteome profiling in Suaeda salsa. J. Plant Physiol. 168, 1743-1752 (2011).

37. Peterson, G. L. A simplification of the protein assay method of Lowry et al. which is more generally applicable. Anal. Biochem. 83, 346-356 (1977).

38. Koehler, G. et al. Proteomic study of low-temperature responses in strawberry cultivars (Fragaria $\times$ ananassa) that differ in cold tolerance. Plant Physiol. 159, 1787-1805 (2012). 
39. Sheffield, J., Taylor, N., Fauquet, C. \& Chen, S. The cassava (Manihot esculenta Crantz) root proteome: Protein identification and differential expression. Proteomics 6, 1588-1598 (2006).

40. Liu, H. M., Fang, L., Che, Y. S., Wu, F. Z. \& Yang, C. P. Protein expression patterns in two Spiraea species in response to cold treatment. Mol. Biol. Rep. 41, 4533-4547 (2014).

41. Ye, Y., Li, X. \& Zhao, J. Production and Characteristics of a Novel Xylose- A nd Alkali-tolerant GH $43 \beta$ xylosidase from Penicillium oxalicum for Promoting Hemicellulose Degradation. Sci. Rep. 7, 1-11 (2017).

42. Wang, X. et al. A cytoplasmic protein Ssl3829 is important for NDH-1 hydrophilic arm assembly in Synechocystis sp. strain PCC 6803. Plant Physiol. 171, 864-877 (2016).

43. Jiang, Y., Yang, B., Harris, N. S. \& Deyholos, M. K. Comparative proteomic analysis of NaCl stressresponsive proteins in Arabidopsis roots. J. Exp. Bot. 58, 3591-3607 (2007).

44. Finn, R. D. et al. The Pfam protein families database: Towards a more sustainable future. Nucleic Acids Res. 44, D279-D285 (2016).

45. Cheng, F. et al. BRAD, the genetics and genomics database for Brassica plants. BMC Plant Biol. 11, 136 (2011).

46. Mistry, J., Finn, R. D., Eddy, S. R., Bateman, A. \& Punta, M. Challenges in homology search: HMMER3 and convergent evolution of coiled-coil regions. Nucleic Acids Res. 41, e121 (2013).

47. Letunic, I., Doerks, T. \& Bork, P. SMART: Recent updates, new developments and status in 2015. Nucleic Acids Res. 43, D257-D260 (2015).

48. Saha, G., Park, J. I., Kayum, M. A. \& Nou, I. S. A genome-wide analysis reveals stress and hormone responsive patterns of TIFY family genes in Brassica rapa. Front. Plant Sci. 7, 1-18 (2016).

49. Lu, K. et al. A combination of genome-wide association and transcriptome analysis reveals candidate genes controlling harvest index-related traits in Brassica napus. Sci. Rep. 6, 1-13 (2016).

50. Marchler-Bauer, A. et al. CDD/SPARCLE: Functional classification of proteins via subfamily domain architectures. Nucleic Acids Res. 45, D200-D203 (2017).

51. Gasteiger, E. et al. Protein Identification and Analysis Tools on the ExPASy Server. The Proteomics Protocols Handbook, Humana Press (2005). doi:10.1385/1-59259-584-7:531

52. Bailey, T. L. et al. MEME Suite: Tools for motif discovery and searching. Nucleic Acids Res. 37, W202-W208 (2009).

53. Hu, B. et al. GSDS 2.0: An upgraded gene feature visualization server. Bioinformatics 31, 1296-1297 (2015).

54. Sievers, F. et al. Fast, scalable generation of high-quality protein multiple sequence alignments using Clustal Omega. Mol. Syst. Biol. 7, 539 (2011).

55. Kumar, S., Stecher, G. \& Tamura, K. MEGA7: Molecular Evolutionary Genetics Analysis Version 7.0 for Bigger Datasets. Mol. Biol. Evol. 33, 1870-1874 (2016).

56. Saitou, N. \& Nei, M. The neighbor-joining method: a new method for reconstructing phylogenetic trees. Mol. Biol. Evol. 4, 406-425 (1987). 
57. Voorrips, R. E. Mapchart: Software for the graphical presentation of linkage maps and QTLs. Journal of Heredity 93, 77-78 (2002).

58. Krzywinski, M. et al. Circos: An information aesthetic for comparative genomics. Genome Res. 19, 1639-1645 (2009).

59. Wang, Y. et al. MCScanX: A toolkit for detection and evolutionary analysis of gene synteny and collinearity. Nucleic Acids Res. 40, e49 (2012).

60. Wang, D., Zhang, Y., Zhang, Z., Zhu, J. \& Yu, J. KaKs_Calculator 2.0: A Toolkit Incorporating GammaSeries Methods and Sliding Window Strategies. Genomics, Proteomics Bioinforma. 8, 77-80 (2010).

61. Livak, K. J. \& Schmittgen, T. D. Analysis of relative gene expression data using real-time quantitative PCR and the 2- $\triangle \Delta C T$ method. Methods 25, 402-408 (2001).

62. Giulietti, A. et al. An overview of real-time quantitative PCR: Applications to quantify cytokine gene expression. Methods 25, 386-401 (2001).

63. Ma, L. et al. Transcriptome Analysis Reveals Key Cold-Stress-Responsive Genes in Winter Rapeseed ( Brassica rapa L .). Int. J. Mol. Sci. 20, 1071 (2019).

64. Pu, Y. et al. Transcriptome profile analysis of winter rapeseed (Brassica napus L.) in response to freezing stress, reveal potentially connected events to freezing stress. Int. J. Mol. Sci. 20, 2771 (2019).

65. Qi, W. et al. Physiological and Biochemical Mechanisms and Cytology of Cold Tolerance in Brassica napus. Front. Plant Sci. 11, 1241 (2020).

66. Liu, Z. et al. Genome-wide identification and analysis of the Q-type $\mathrm{C} 2 \mathrm{H} 2$ gene family in potato (Solanum tuberosum L.). Int. J. Biol. Macromol. 153, 327-340 (2020).

67. Xiaochuang, C. et al. Glycine increases cold tolerance in rice via the regulation of $\mathrm{N}$ uptake, physiological characteristics, and photosynthesis. Plant Physiol. Biochem. 112, 251-260 (2017).

68. Tao, C. et al. Genome-wide investigation and expression profiling of APX gene family in Gossypium hirsutum provide new insights in redox homeostasis maintenance during different fiber development stages. Mol. Genet. Genomics 293, 685-697 (2018).

69. Asada, K. The water-water cycle in chloroplasts: Scavenging of active oxygens and dissipation of excess photons. Annu. Rev. Plant Biol. 50, 601-639 (1999).

70. Teixeira, F. K., Menezes-Benavente, L., Galvão, V. C., Margis, R. \& Margis-Pinheiro, M. Rice ascorbate peroxidase gene family encodes functionally diverse isoforms localized in different subcellular compartments. Planta 224, 300-314 (2006).

71. Teixeira, F. K., Menezes-Benavente, L., Margis, R. \& Margis-Pinheiro, M. Analysis of the molecular evolutionary history of the ascorbate peroxidase gene family: Inferences from the rice genome. $J$. Mol. Evol. 59, 761-770 (2004).

72. Gupta, S., Dong, Y., Dijkwel, P. P., Mueller-Roeber, B. \& Gechev, T. S. Genome-wide analysis of ROS antioxidant genes in resurrection species suggest an involvement of distinct ROS detoxification systems during desiccation. Int. J. Mol. Sci. 20, 3101 (2019). 
73. Wei, L. et al. Genome wide identification and comparative analysis of glutathione transferases (GST) family genes in Brassica napus. Sci. Rep. 9, 9196 (2019).

74. Li, Y. et al. Genome-wide analysis and expression profiles of the StR2R3-MYB transcription factor superfamily in potato (Solanum tuberosum L.). Int. J. Biol. Macromol. 148, 817-832 (2020).

75. Dias, A. P., Braun, E. L., McMullen, M. D. \& Grotewold, E. Recently duplicated maize R2R3 Myb genes provide evidence for distinct mechanisms of evolutionary divergence after duplication. Plant Physiol. 131, 610-620 (2003).

76. Guan, Q., Wang, Z., Wang, X., Takano, T. \& Liu, S. A peroxisomal APX from Puccinellia tenuiflora improves the abiotic stress tolerance of transgenic Arabidopsis thaliana through decreasing of H2O2accumulation. J. Plant Physiol. 175, 183-191 (2015).

77. Panchuk, I. I., Zentgraf, U. \& Volkov, R. A. Expression of the Apx gene family during leaf senescence of Arabidopsis thaliana. Planta 222, 926-932 (2005).

78. Verma, D., Lakhanpal, N. \& Singh, K. Genome-wide identification and characterization of abioticstress responsive SOD (superoxide dismutase) gene family in Brassica juncea and B. rapa. BMC Genomics 20, 227 (2019).

79. Noctor, G., Mhamdi, A. \& Foyer, C. H. The roles of reactive oxygen metabolism in drought: Not so cut and dried. Plant Physiol. 164, 1636-1648 (2014).

80. Foyer, C. H., Bloom, A. J., Queval, G. \& Noctor, G. Photorespiratory metabolism: Genes, mutants, energetics, and redox signaling. Annu. Rev. Plant Biol. 60, 455-484 (2009).

81. Shafi, A. et al. Transgenic Potato Plants Overexpressing SOD and APX Exhibit Enhanced Lignification and Starch Biosynthesis with Improved Salt Stress Tolerance. Plant Mol. Biol. Report. 35, 504-518 (2017).

82. Yan, H. et al. Overexpression of CuZnSOD and APX enhance salt stress tolerance in sweet potato. Plant Physiol. Biochem. 109, 20-27 (2016).

83. Zhou, F. et al. Genome-wide analysis of mdhar gene family in four cotton species provides insights into fiber development via regulating asa redox homeostasis. Plants 10, 227 (2021).

84. Cao, Y. et al. Exogenous sucrose increases chilling tolerance in cucumber seedlings by modulating antioxidant enzyme activity and regulating proline and soluble sugar contents. Sci. Hortic. (Amsterdam). 179, 67-77 (2014).

85. Yan, S., Zhang, Q., Tang, Z., Su, W. \& Sun, W. Comparative Proteomic Analysis Provides New Insights into Chilling Stress Responses in Rice. Mol. Cell. Proteomics 5, 484-496 (2006).

86. Jędrzejuk, A., Rabiza-Świder, J., Skutnik, E. \& Łukaszewska, A. Growing conditions and preservatives affect longevity, soluble protein, $\mathrm{H} 2 \mathrm{O} 2$ and MDA contents, activity of antioxidant enzymes and DNA degradation in cut lilacs. Sci. Hortic. (Amsterdam). 228, 122-131 (2018).

87. Tian, X. et al. Comparative proteomic analysis of seedling leaves of cold-tolerant and -sensitive spring soybean cultivars. Mol. Biol. Rep. 42, 581-601 (2015). 
88. O’Kane, D., Gill, V., Boyd, P. \& Burdon, R. Chilling, oxidative stress and antioxidant responses in Arabidopsis thaliana callus. Planta 198, 371-377 (1996).

89. Zhang, B. Q., Yang, L. T. \& Li, Y. R. Physiological and Biochemical Characteristics Related to Cold Resistance in Sugarcane. Sugar Tech 17, 49-58 (2015).

90. Ghorbanpour, A. et al. The effect of Trichoderma harzianum in mitigating low temperature stress in tomato (Solanum lycopersicum L.) plants. Sci. Hortic. (Amsterdam). 230, 134-141 (2018).

91. Szymańska, R., Ślesak, I., Orzechowska, A. \& Kruk, J. Physiological and biochemical responses to high light and temperature stress in plants. Environmental and Experimental Botany 139, 165-177 (2017).

92. He, C., Gao, G., Zhang, J., Duan, A. \& Luo, H. Proteome profiling reveals insights into cold-tolerant growth in sea buckthorn. Proteome Sci. 14, 14 (2016).

93. Wang, X., Liu, Y. \& Yang, P. Proteomic studies of the abiotic stresses response in model mossPhyscomitrella patens. Front. Plant Sci. 3, 258 (2012).

94. Zhang, L. et al. Identification of an apoplastic protein involved in the initial phase of salt stress response in rice root by two-dimensional electrophoresis. Plant Physiol. 149, 916-928 (2009).

95. Kong, F. J., Oyanagi, A. \& Komatsu, S. Cell wall proteome of wheat roots under flooding stress using gel-based and LC MS/MS-based proteomics approaches. Biochim. Biophys. Acta - Proteins Proteomics 1804, 124-136 (2010).

96. Rychnovská, M. A. Sakai and W. Larcher Frost survival of plants responses and adaptation to freezing stress. Folia Geobot. Phytotaxon. 24, 252-252 (1989).

97. Rakei, A., Maali-Amiri, R., Zeinali, H. \& Ranjbar, M. DNA methylation and physio-biochemical analysis of chickpea in response to cold stress. Protoplasma 253, 61-76 (2016).

98. Fan, J. et al. Cloning and Function Analysis of Gene SAMS from Glycine soja. Acta Agron. Sin. 34, 1581-1587 (2008).

99. Wang, N., Xiao, B. \& Xiong, L. Identification of a cluster of PR4-like genes involved in stress responses in rice. J. Plant Physiol. 168, 2212-2224 (2011).

100. Seo, P. J. et al. Cold activation of a plasma membrane-tethered NAC transcription factor induces a pathogen resistance response in Arabidopsis. Plant J. 61, 661-671 (2010).

101. Juan, Y. et al. Cold-inducible RNA-binding protein mediates airway inflammation and mucus hypersecretion through a post-transcriptional regulatory mechanism under cold stress. Int. J. Biochem. Cell Biol. 78, 335-348 (2016).

\section{Figures}


A

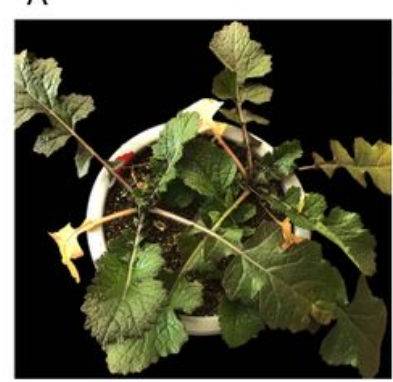

C

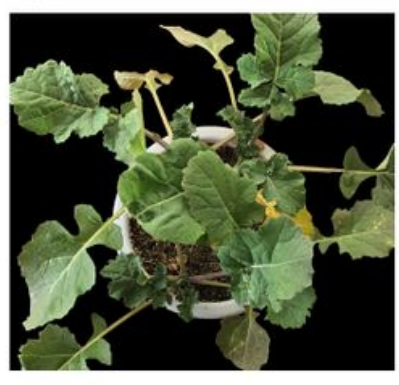

B

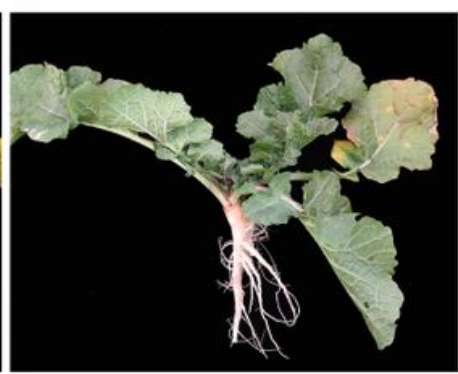

$\mathrm{D}$

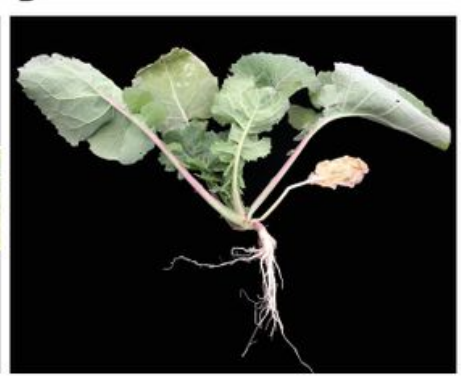

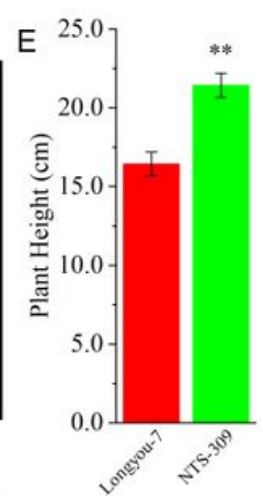

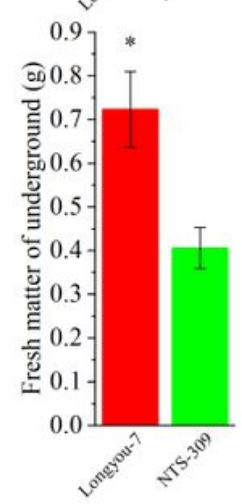

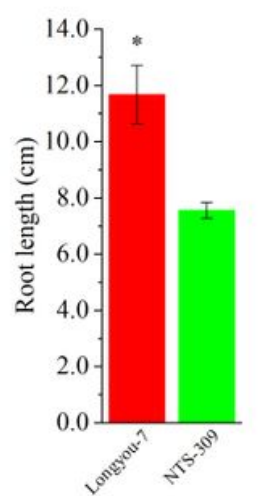
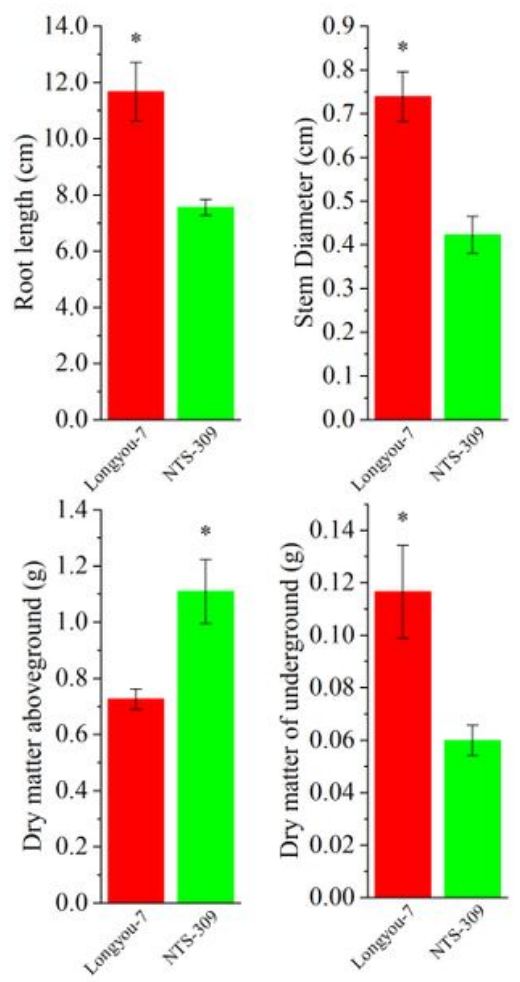
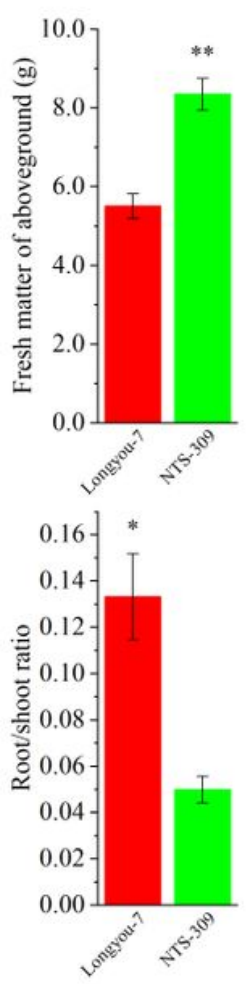

Figure 1

Comparison of morphological characteristics of Longyou-7 and NTS-309. A and B are the growth characteristics of Longyou-7, C and are the growth characteristics of TS-309. Error bars indicate the SD for three biological replicates. * and ** indicate significance at the $\mathrm{P} \leq 0.05$ and 0.01 levels, respectively. 

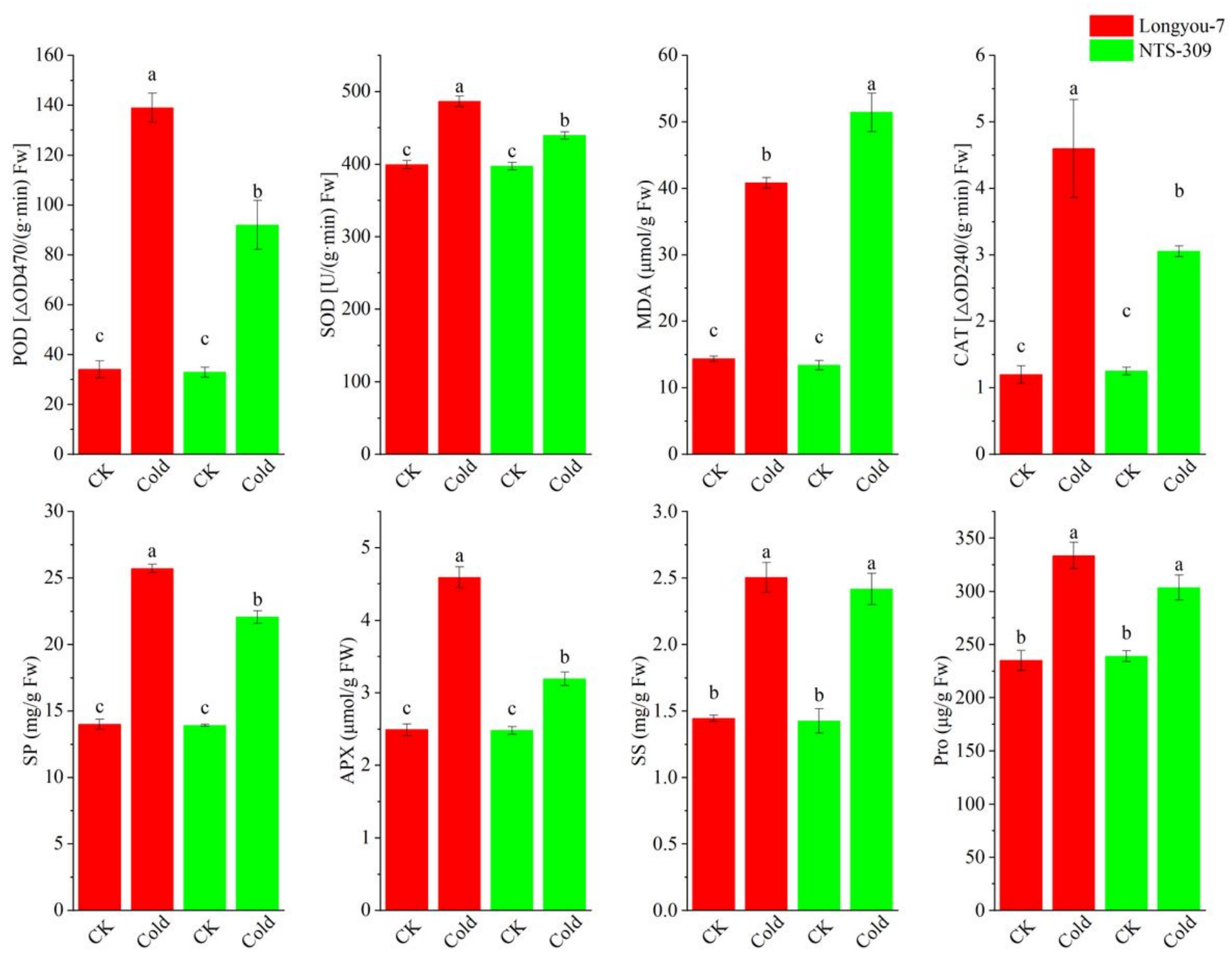

\section{Figure 2}

Comparison of physiological characteristics of winter rapeseed under cold stress. The peroxidase (POD), superoxide dismutase (SOD), malondialdehyde (MDA), catalase (CAT), soluble protein (SP), ascorbate peroxidase (APX), soluble sugar (SS) and free proline (Pro) in growth cone of winter rapeseed were determined under cold stress. Error bars indicate the SD for three biological replicates. 


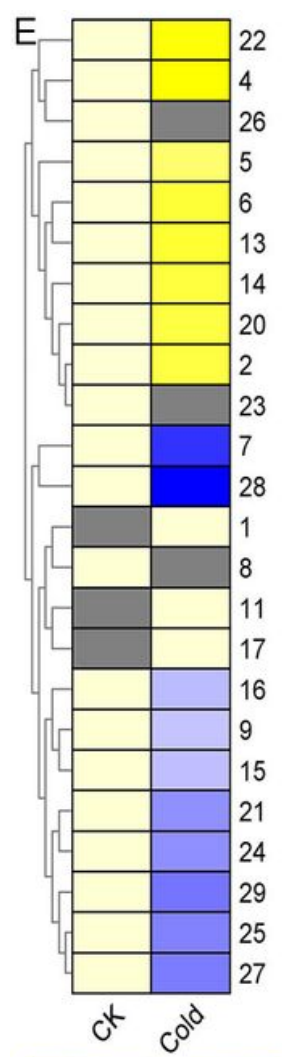

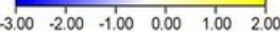
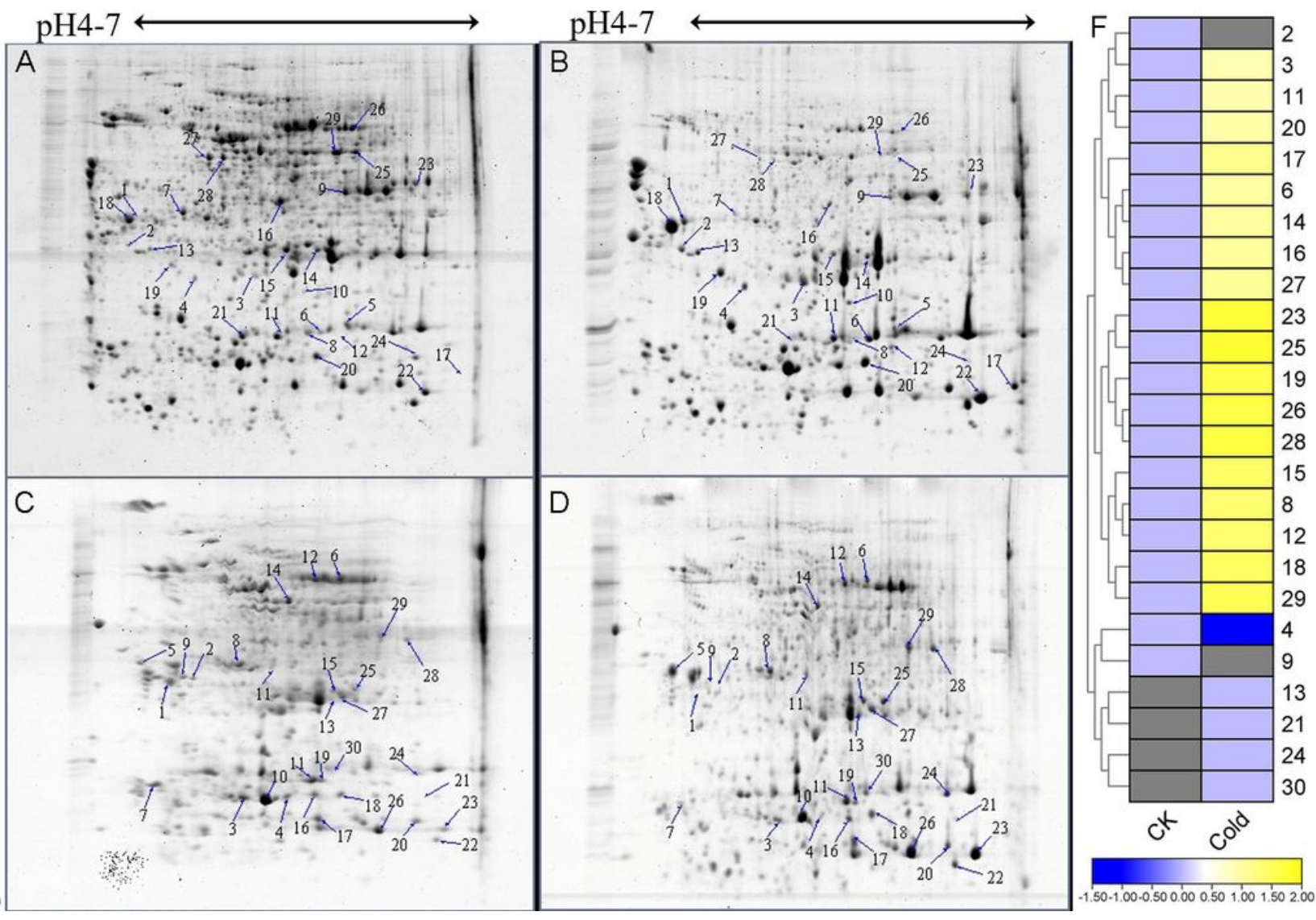

Figure 3

Clustering and expression abundance of differentially expressed proteins under cold stress. Longyou-7 (A, $B, E)$ and NTS-309 (C, D, F) treated with $24^{\circ} \mathrm{C}$ for CK (A, C), $4^{\circ} \mathrm{C}$ for $24 \mathrm{~h}(\mathrm{~B}, \mathrm{D})$. Arrows indicate 59 protein spots that were positively identified $(P \leq 0.05)$. Yellow and blue show Log2-fold up- and down-regulated protein 


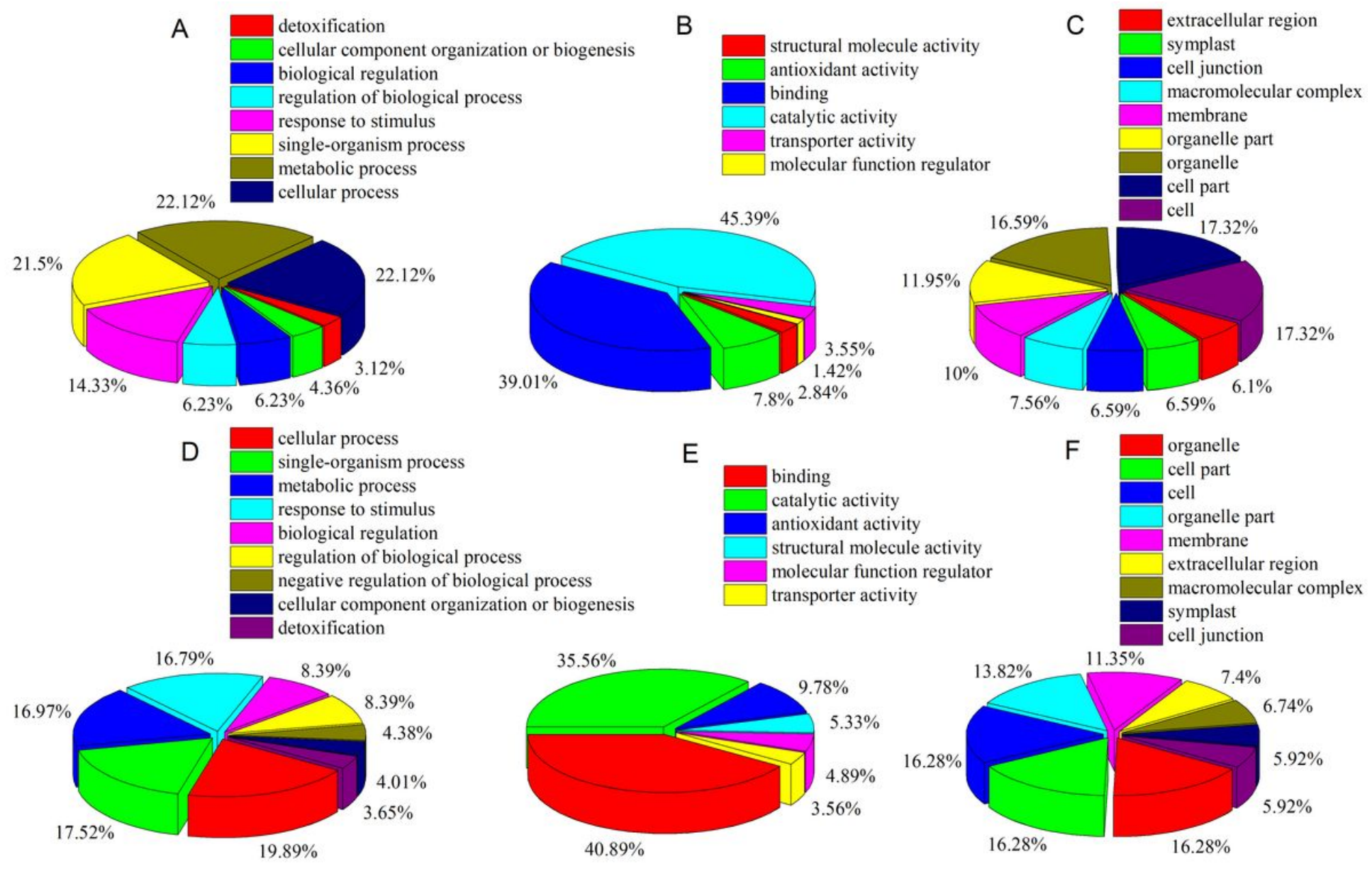

Figure 4

Classification and functional analysis of DEPs in growth cones of Longyou-7(A, B, C) and NTS-309(D, E, F) under cold-stress. Results of the gene ontology (GO) classification and pathway analysis based on cellular component $(A, D)$, molecular function $(B, E)$, and biological process $(C, F)$ are highlighted. 


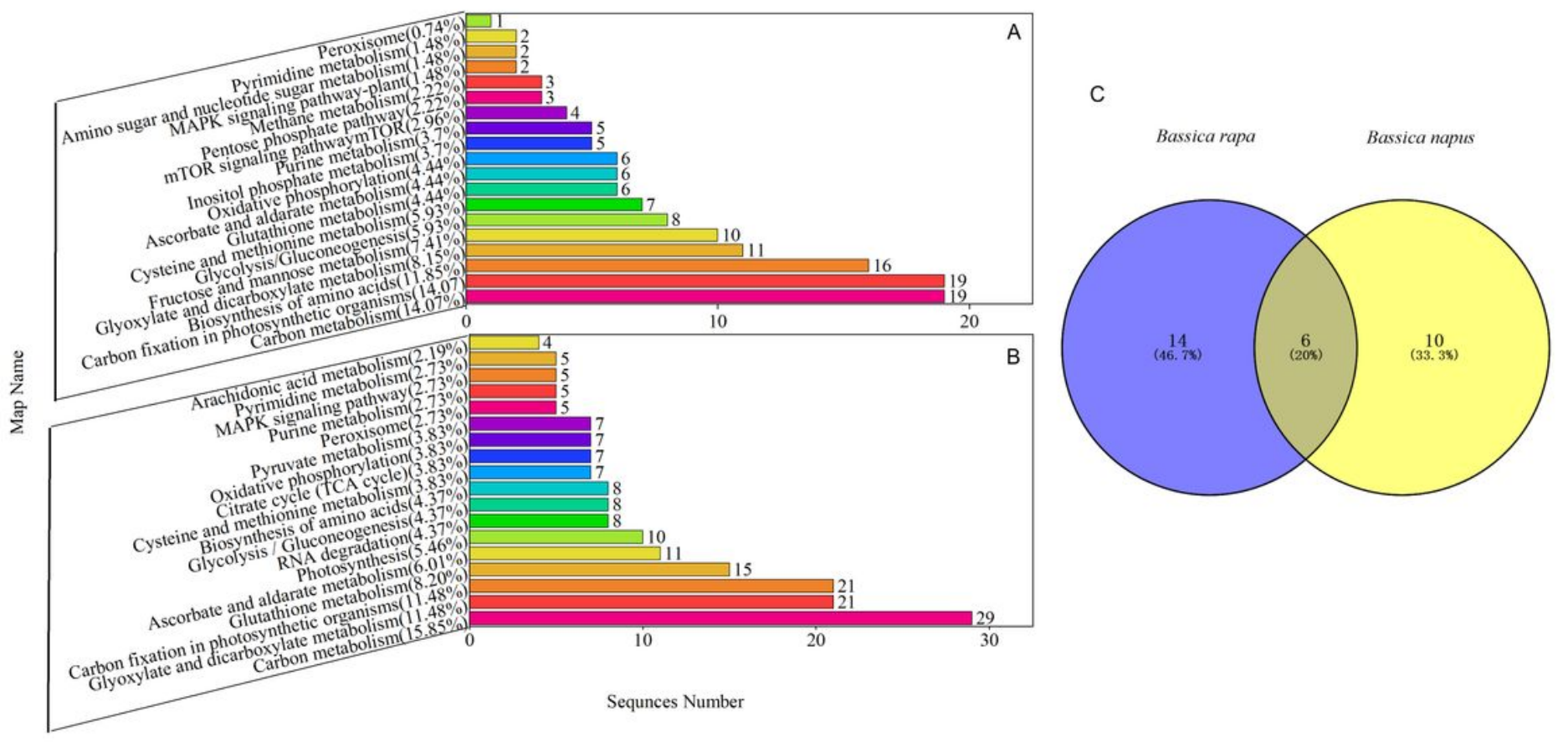

Figure 5

KEGG annotation and Venn diagrams of DEP in growth cone of Longyou-7 and NTS-309 under cold stress. 
A

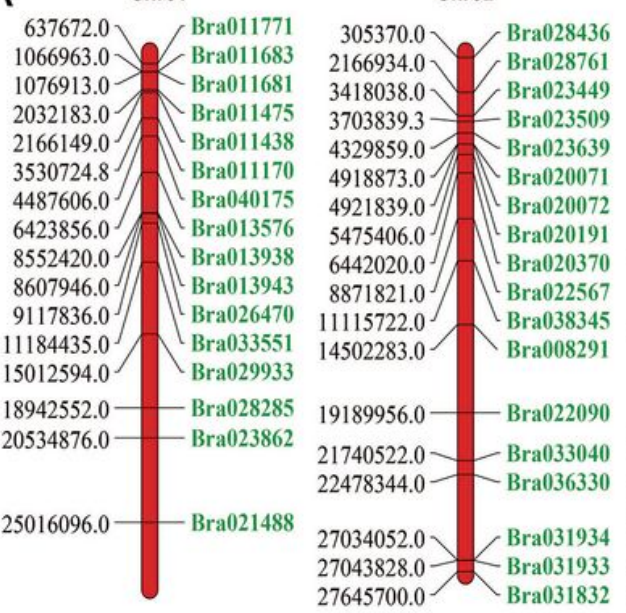

Chro3
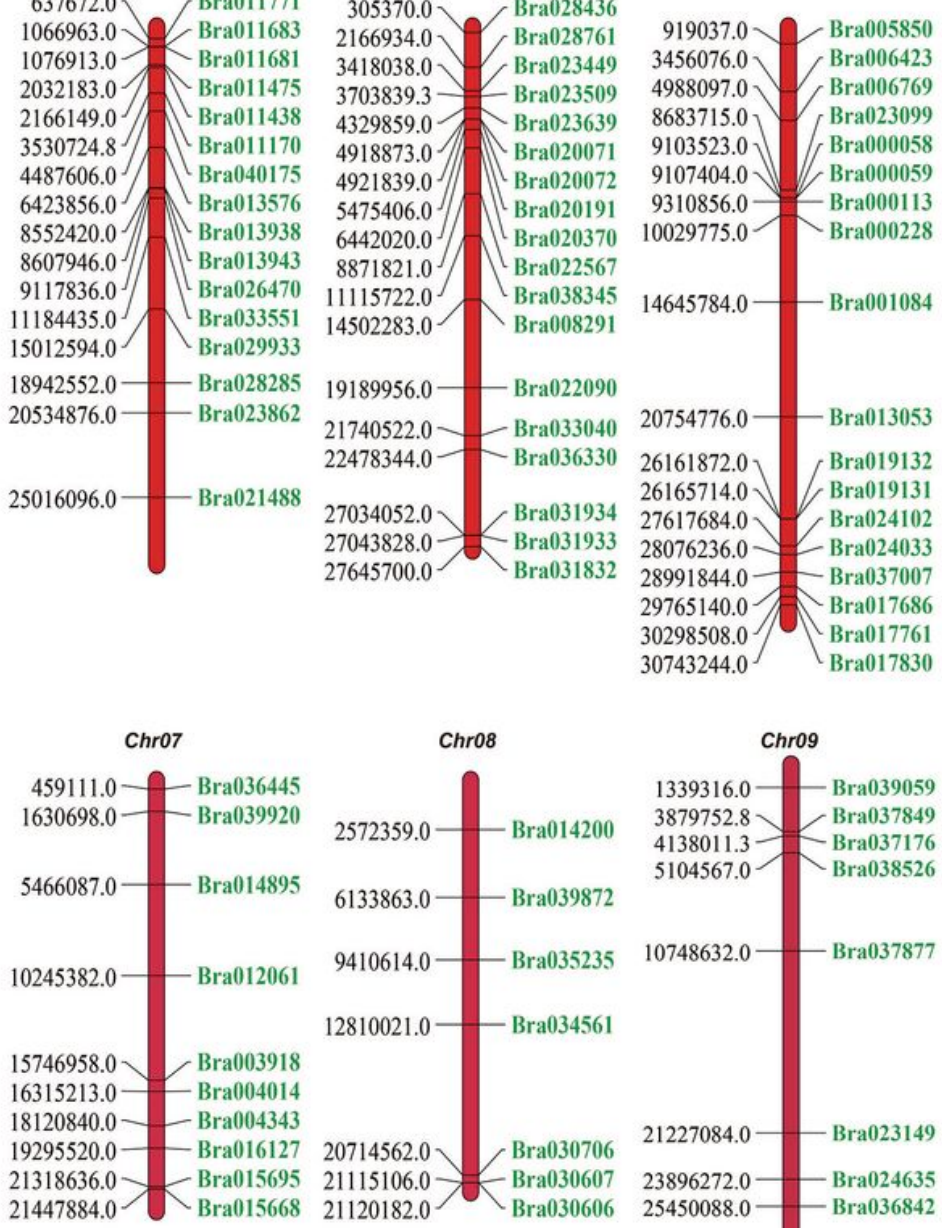

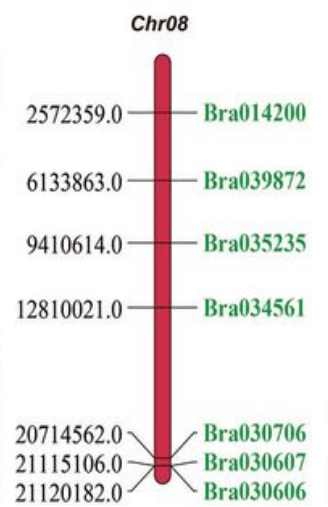

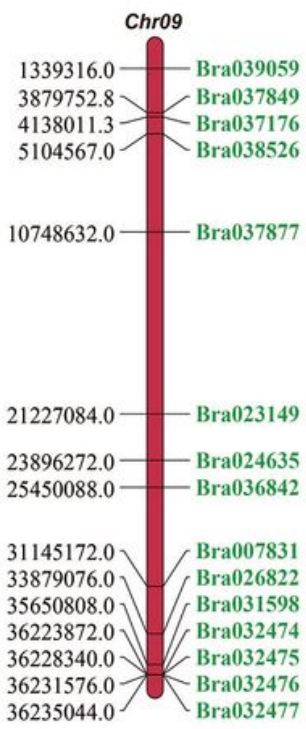

Chro4

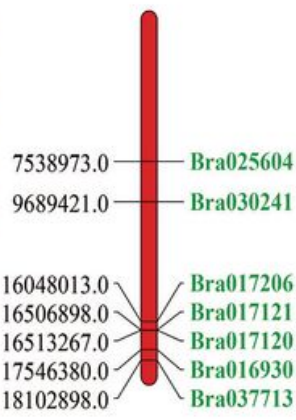

18102898.0 Bra01693

$16118925.0-$ Bra031309

$19171084.0-$ Bra022195
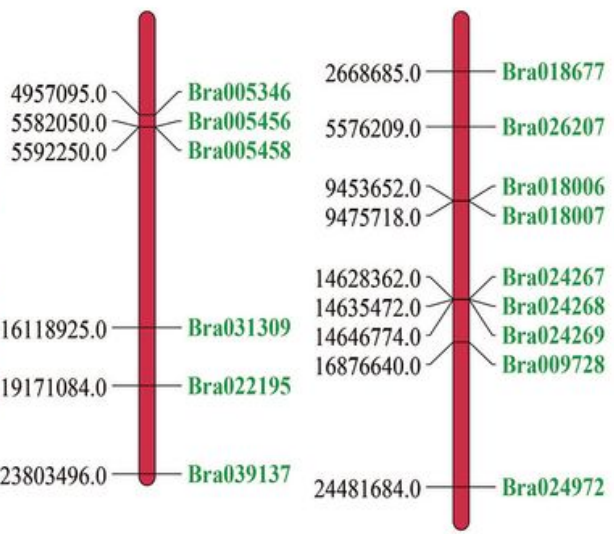

B
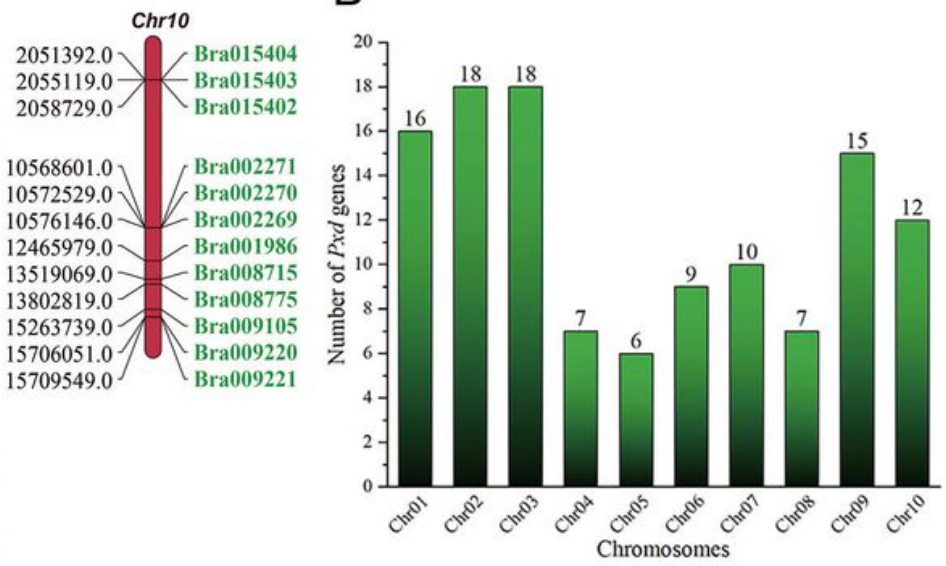

\section{Figure 6}

Chromosome mapping of the APX gene in B. rapa. (A) Map of the distribution of B. rapa APX genes on 10 chromosomes. (B) Number of APX genes on each chromosome. 


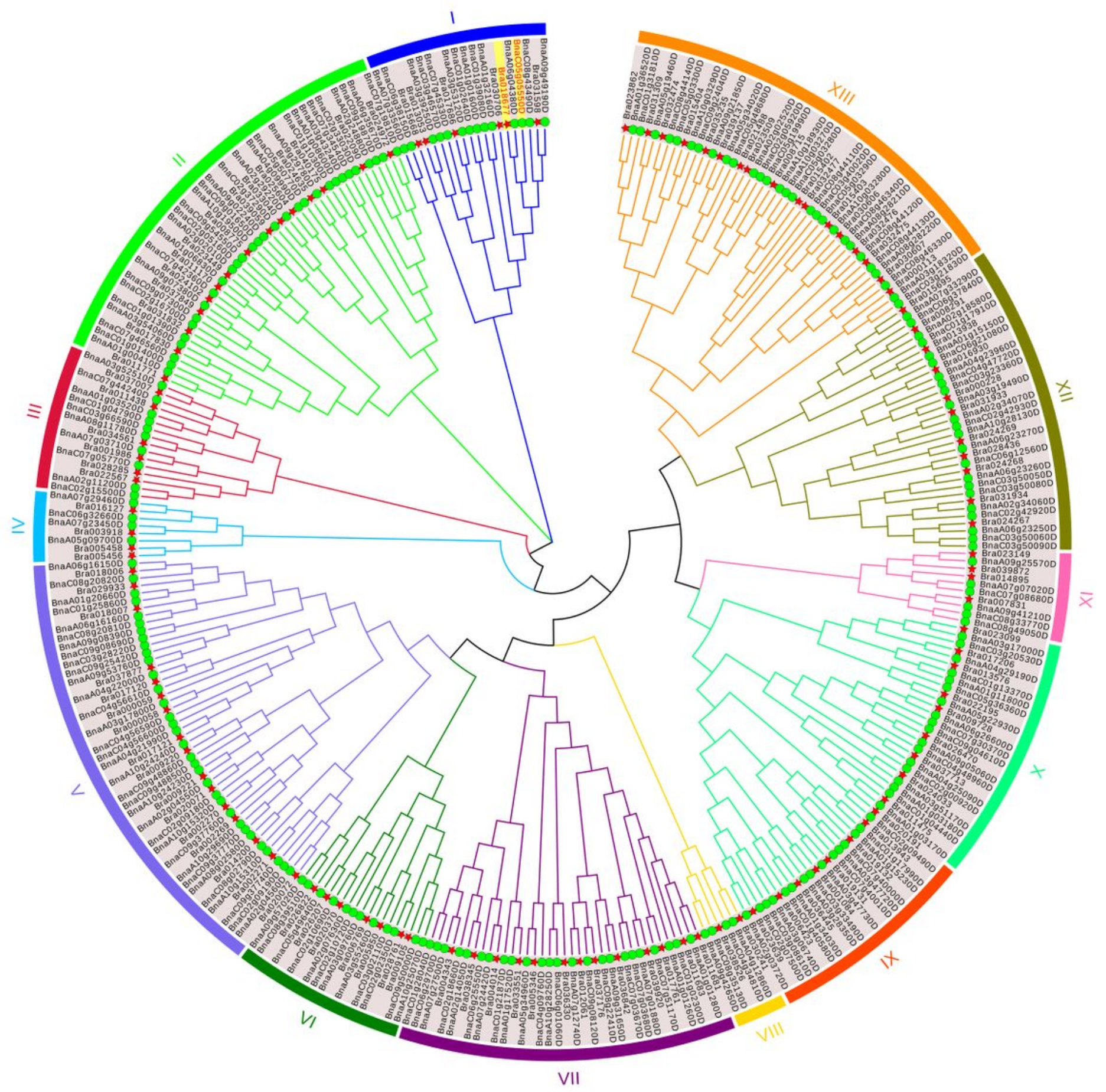

Figure 7

Phylogenetic tree of APX protein of B.napus and B.rapa. 13 subfamilies and branches are marked with different colors. Green circles represent B.napus, red stars represent B.rapa, and two proteins from proteomic identification are marked with red font and yellow background. 

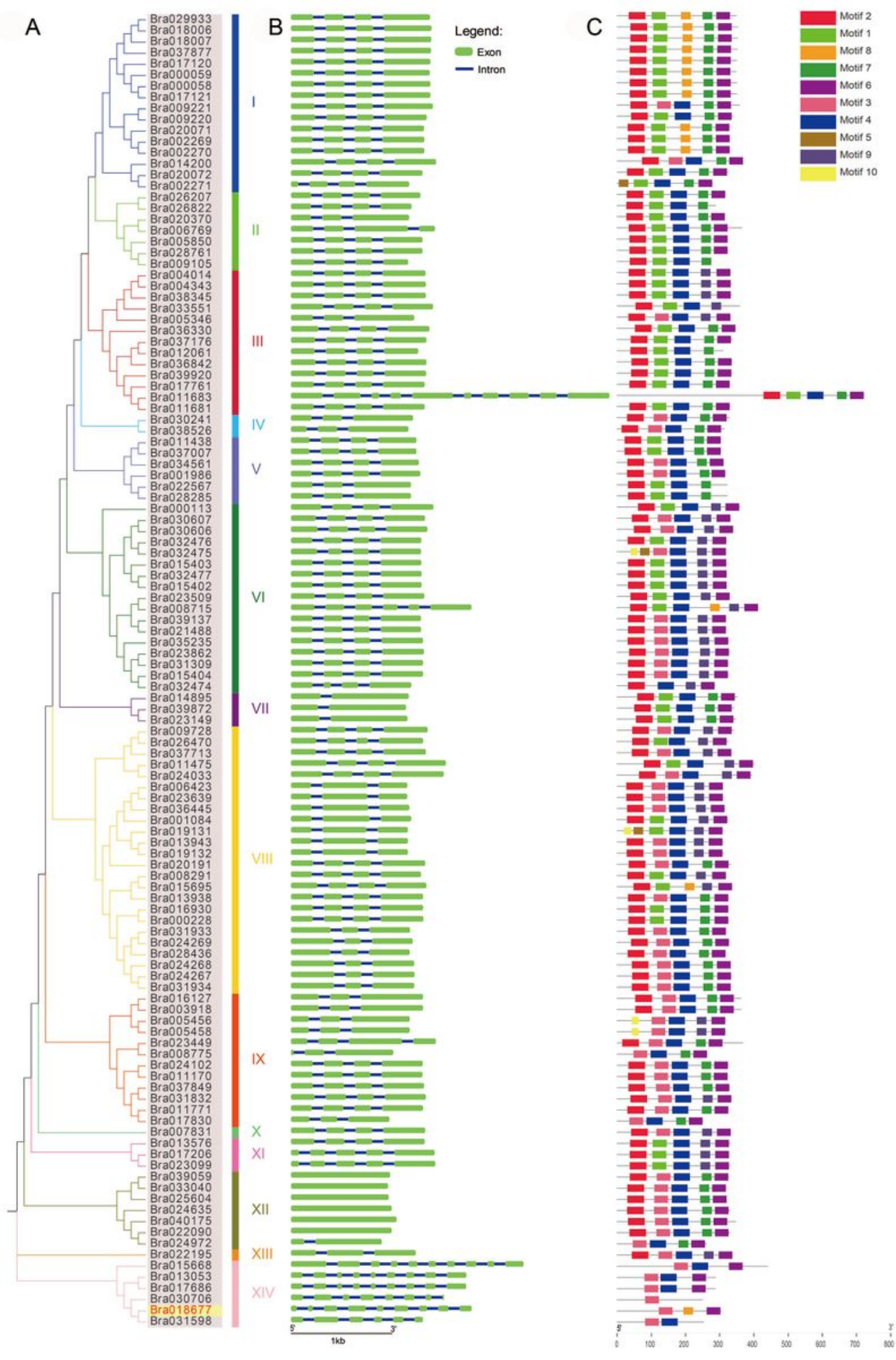

Figure 8

Phylogenetic tree, gene structure and motif analysis of BrAPXs. (A) BrAPXs evolutionary relationships. (B) Structure of intron/exon in BrAPXs, green boxes represent exons and blue lines represent introns. (C) Conserved motif distribution of BrAPXs, different color boxes represent 10 conserved domains identified. 

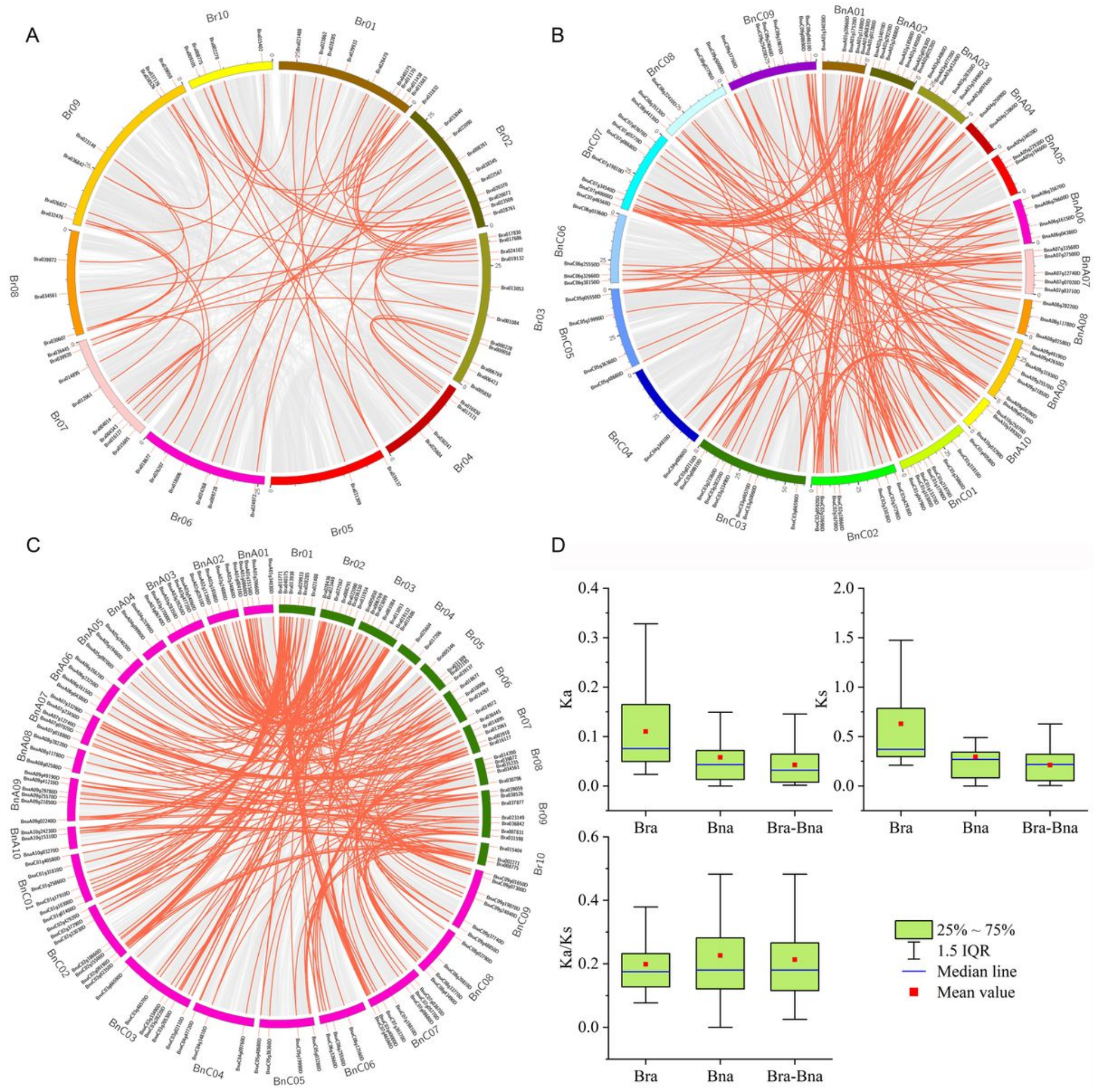

Figure 9

APX segmental duplicated gene pairs in B. rapa genome (A), APX segmental duplicated gene pairs in $B$. napus genome (B) and homology of APX in B. rapa and B.napus (C), the red lines in A-C indicate the segmental duplicated gene pairs and the chromosome numbers are shown at the top of each chromosome. The mean values of $\mathrm{Ka}, \mathrm{Ks}$ and $\mathrm{Ka} / \mathrm{Ks}$ for duplicated genes are shown in $\mathrm{D}$. 

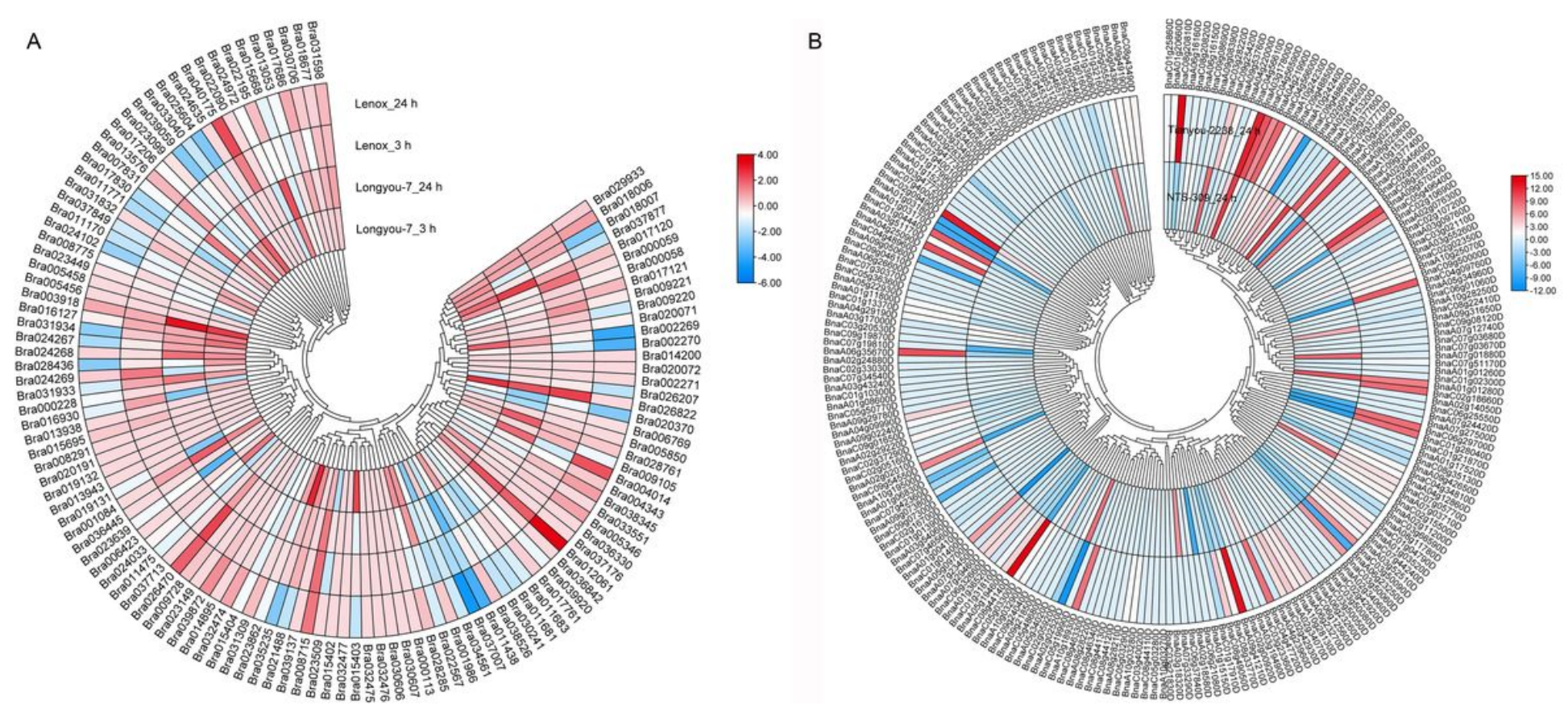

Figure 10

The expression heatmap of BrAPXs (A) and BnAPXs (B) of different cold-resistant varieties under cold stress was plotted according to the log2 mean of FPKM in RNA-seq. 

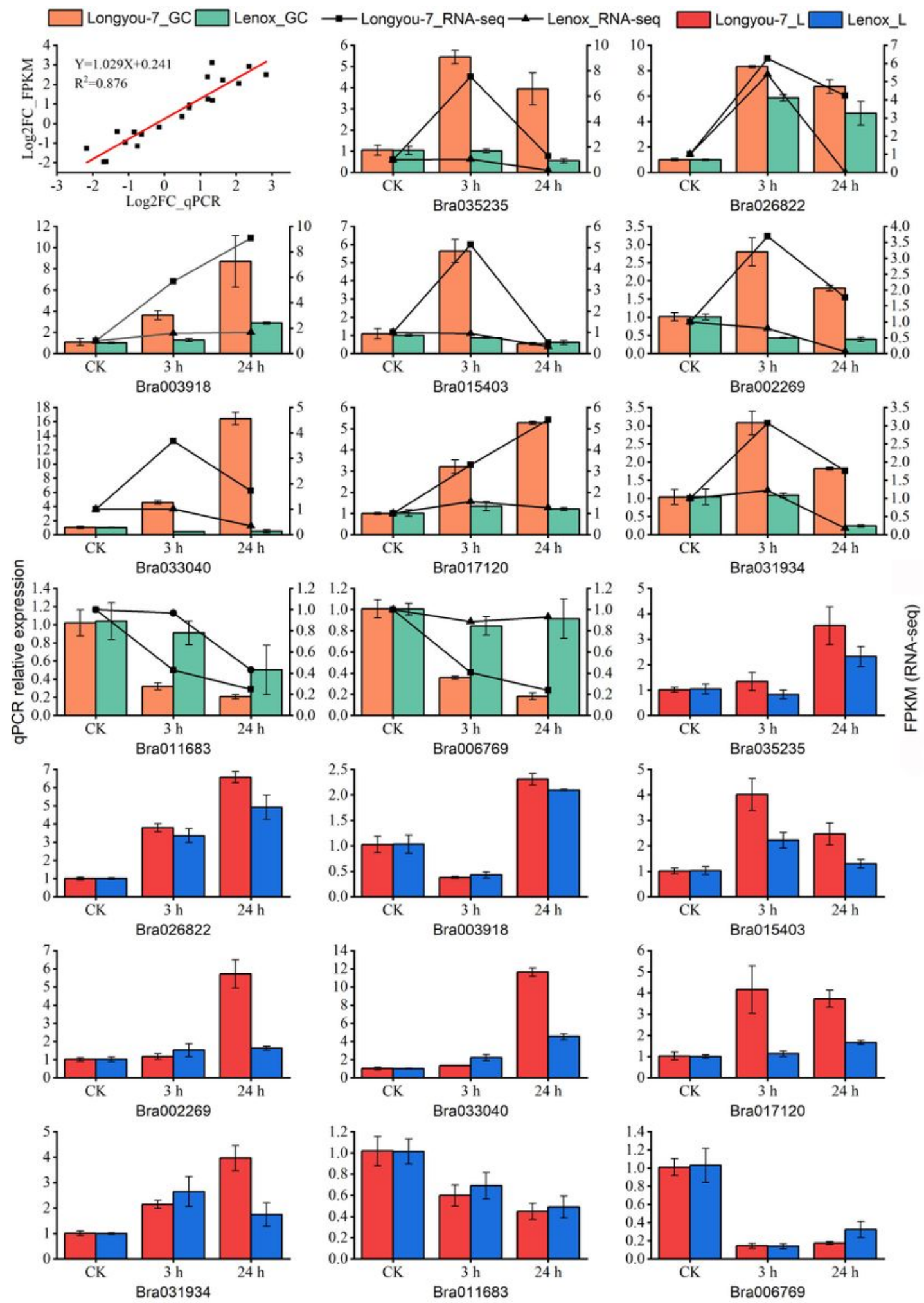

Figure 11

10 APX genes were analyzed by qPCR expression in growth cones (GC) and leaves (L) of cold-tolerant (Longyou-7) and cold-sensitive varieties (Lenox) under cold stress treatment. Gene expression was normalized to the expression level of normal growth at room temperature and assigned a value of 1 . Data represent the mean \pm standard error for three biological experiments, with standard errors shown as bar charts above the columns. qPCR and RNA-seq correlations at the Log2 level are also shown. 


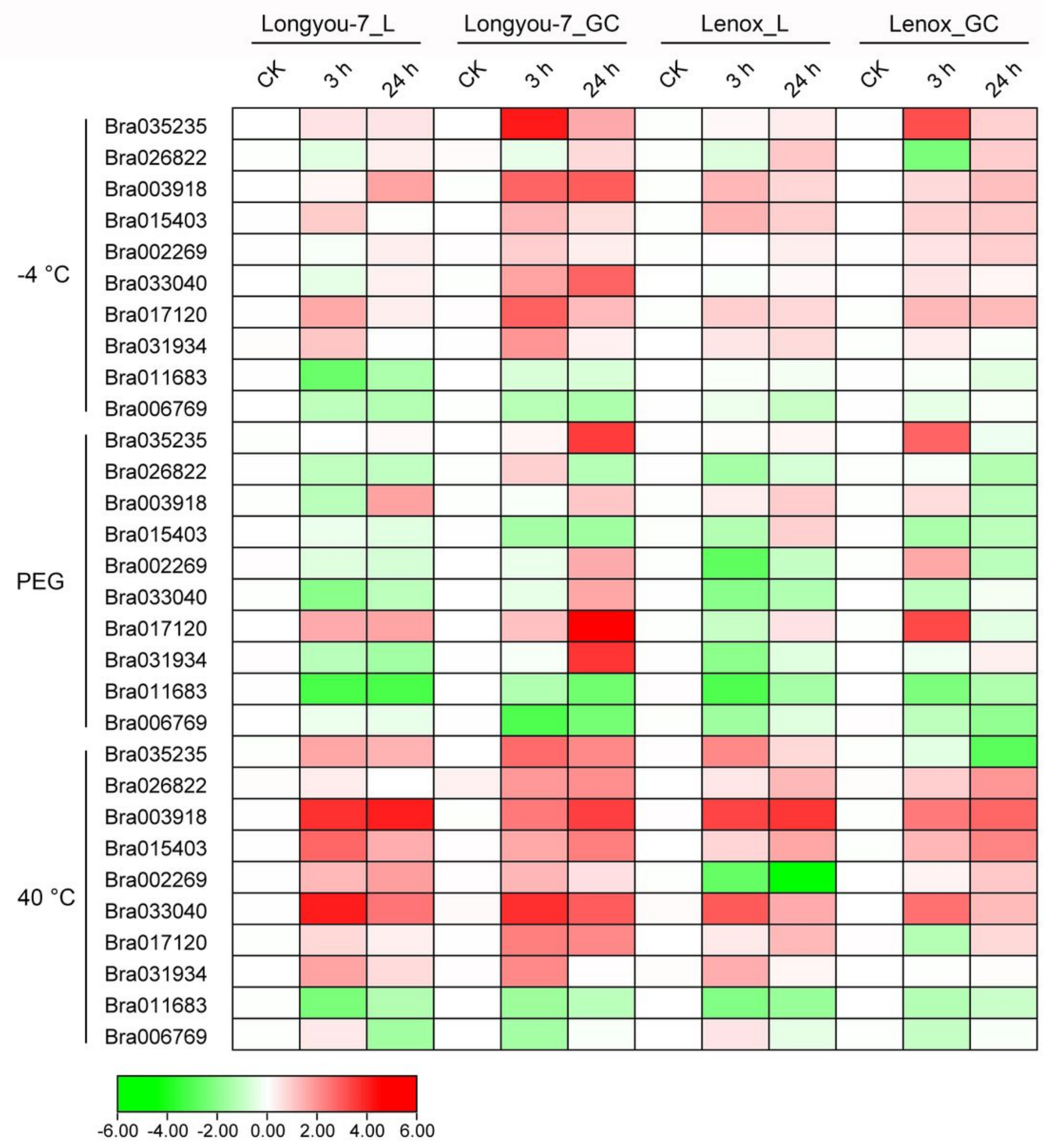

\section{Figure 12}

The expression profiles of 10 APX genes in Longyou-7 and Lenox growth cones (GC) and leaves (L) under freezing, drought and heat stress treatments for $3 \mathrm{~h}$ and $24 \mathrm{~h}$. Gene expression was normalized to the expression level of normal growth and assigned a value of 1. Data represent Log2 of the mean of three biological experiments. 


\section{Supplementary Files}

This is a list of supplementary files associated with this preprint. Click to download.

- Supplementaryfile1TableS1.docx

- Supplementaryfile2FigureS12Dgels6.zip

- Supplementaryfile3TableS2proteinsofLongyou7.docx

- Supplementaryfile4TableS3IdentificationLongyou7.xlsx

- Supplementaryfile5TableS4proteinsofTS309.docx

- Supplementaryfile6TableS5IdentificationNTS309.xIsx

- Supplementaryfile7TableS6Basicphysicochemical.xlsx

- Supplementaryfile8TableS7Motifprediction.xIsx

- Supplementaryfile9TableS8Thegeneduplicationevents.xlsx

- Supplementaryfile10TableS9Log2meansofFPKM.xlsx 